\title{
The Generating Function of Ternary Trees and Continued Fractions
}

\author{
Ira M. Gessel* and Guoce Xin ${ }^{\dagger}$ \\ Department of Mathematics \\ Brandeis University \\ Waltham MA 02454-9110 \\ gessel@brandeis.edu \\ Department of Mathematics \\ Brandeis University \\ Waltham MA 02454-9110 \\ guoce.xin@gmail.com
}

Submitted: May 4, 2005; Accepted: Feb 1, 2006; Published: Jun 12, 2006

Mathematics Subject Classification: 05A15; 05A10, 05A17, 30B70, 33C05

\begin{abstract}
Michael Somos conjectured a relation between Hankel determinants whose entries $\frac{1}{2 n+1}\left(\begin{array}{c}3 n \\ n\end{array}\right)$ count ternary trees and the number of certain plane partitions and alternating sign matrices. Tamm evaluated these determinants by showing that the generating function for these entries has a continued fraction that is a special case of Gauss's continued fraction for a quotient of hypergeometric series. We give a systematic application of the continued fraction method to a number of similar Hankel determinants. We also describe a simple method for transforming determinants using the generating function for their entries. In this way we transform Somos's Hankel determinants to known determinants, and we obtain, up to a power of 3 , a Hankel determinant for the number of alternating sign matrices. We obtain a combinatorial proof, in terms of nonintersecting paths, of determinant identities involving the number of ternary trees and more general determinant identities involving the number of $r$-ary trees.
\end{abstract}

\footnotetext{
*Partially supported by NSF Grant DMS-0200596.

${ }^{\dagger}$ Both authors wish to thank the Institut Mittag-Leffler and the organizers of the Algebraic Combinatorics program held there in the spring of 2005, Anders Björner and Richard Stanley.
} 


\section{Introduction}

Let $a_{n}=\frac{1}{2 n+1}\left(\begin{array}{c}3 n \\ n\end{array}\right)=\frac{1}{3 n+1}\left(\begin{array}{c}3 n+1 \\ n\end{array}\right)$ be the number of ternary trees with $n$ vertices and define the Hankel determinants

$$
\begin{aligned}
U_{n} & =\operatorname{det}\left(a_{i+j}\right)_{0 \leq i, j \leq n-1} \\
V_{n} & =\operatorname{det}\left(a_{i+j+1}\right)_{0 \leq i, j \leq n-1} \\
W_{n} & =\operatorname{det}\left(a_{(i+j+1) / 2}\right)_{0 \leq i, j \leq n-1},
\end{aligned}
$$

where we take $a_{k}$ to be 0 if $k$ is not an integer. (We also interpret determinants of $0 \times 0$ matrices as 1.) The first few values of these determinants are

\begin{tabular}{r|rrrrrrr}
$n$ & 1 & 2 & 3 & \multicolumn{1}{c}{4} & \multicolumn{1}{c}{5} & \multicolumn{1}{c}{6} & \multicolumn{1}{c}{7} \\
\hline$U_{n}$ & 1 & 2 & 11 & 170 & 7429 & 920460 & 323801820 \\
$V_{n}$ & 1 & 3 & 26 & 646 & 45885 & 9304650 & 5382618660 \\
$W_{n}$ & 1 & 1 & 2 & 6 & 33 & 286 & 4420
\end{tabular}

This paper began as an attempt to prove the conjectures of Michael Somos [27] that

(a) $U_{n}$ is the number of of cyclically symmetric transpose complement plane partitions whose Ferrers diagrams fit in an $n \times n \times n$ box,

(b) $V_{n}$ is the number of $(2 n+1) \times(2 n+1)$ alternating sign matrices that are invariant under vertical reflection, and

(c) $W_{n}$ is the number of $(2 n+1) \times(2 n+1)$ alternating sign matrices that are invariant under both vertical and horizontal reflection.

Mills, Robbins, and Rumsey [22] (see also [5, Eq. (6.15), p. 199]) showed that the number of objects of type (a) is

$$
\prod_{i=1}^{n-1} \frac{(3 i+1)(6 i) !(2 i) !}{(4 i+1) !(4 i) !}
$$

Mills [25] conjectured the formula

$$
\prod_{i=1}^{n} \frac{\left(\begin{array}{c}
6 i-2 \\
2 i
\end{array}\right)}{2\left(\begin{array}{c}
4 i-1 \\
2 i
\end{array}\right)}
$$

for objects of type (b) and this conjecture was proved by Kuperberg [19]. A formula for objects of type (c) was conjectured by Robbins [26] and proved by Okada [23]. A determinant formula for these objects was proved by Kuperberg [19].

It turns out that it is much easier to evaluate Somos's determinants than to relate them directly to (a)-(c). It is easy to see that $W_{2 n}=U_{n} V_{n}$ and $W_{2 n+1}=U_{n+1} V_{n}$, so it is only necessary show that $U_{n}$ is equal to (4) and $V_{n}$ is equal to (5) to prove Somos's conjectures. 
This was done by Tamm [28], who was unaware of Somos's conjectures. Thus Somos's conjectures are already proved; nevertheless, our study of these conjecture led to some additional determinant evaluations and transformations that are the subject of this paper.

Tamm's proof used the fact that Hankel determinants can be evaluated using continued fractions; the continued fraction that gives these Hankel determinants is a special case of Gauss's continued fraction for a quotient of hypergeometric series. The determinant $V_{n}$ was also evaluated, using a different method, by Eğecioğlu, Redmond, and Ryavec [6, Theorem 4], who also noted the connection with alternating sign matrices and gave several additional Hankel determinants for $V_{n}$ :

$$
V_{n}=\operatorname{det}\left(b_{i+j}\right)_{0 \leq i, j \leq n-1}=\operatorname{det}\left(r_{i+j}\right)_{0 \leq i, j \leq n-1}=\operatorname{det}\left(s_{i+j}(u)\right)_{0 \leq i, j \leq n-1},
$$

where $b_{n}=\frac{1}{n+1}\left(\begin{array}{c}3 n+1 \\ n\end{array}\right), r_{n}=\left(\begin{array}{c}3 n+2 \\ n\end{array}\right)$, and

$$
s_{n}(u)=\sum_{k=0}^{n} \frac{k+1}{n+1}\left(\begin{array}{c}
3 n-k+1 \\
n-k
\end{array}\right) u^{k},
$$

where $u$ is arbitrary. As noted in [6, Theorem 4], $s_{n}(0)=b_{n}, s_{n}(1)=a_{n+1}$, and $s_{n}(3)=r_{n}$.

In Section 2, we describe Tamm's continued fraction method for evaluating these determinants. In Section 3, we give a systematic application of the continued fraction method to several similar Hankel determinants. In Theorem 3.1 we give five pairs of generating functions similar to that for $a_{n}$ whose continued fractions are instances of Gauss's theorem. Three of them have known combinatorial meanings for their coefficients, including the number of two-stack-sortable permutations (see West [29]).

In Section 4 we discuss a simple method, using generating functions, for transforming determinants and use it to show that

$$
U_{n}=\operatorname{det}\left(\left(\begin{array}{c}
i+j \\
2 i-j
\end{array}\right)\right)_{0 \leq i, j \leq n-1}
$$

and

$$
V_{n}=\operatorname{det}\left(\left(\begin{array}{c}
i+j+1 \\
2 i-j
\end{array}\right)\right)_{0 \leq i, j \leq n-1} .
$$

We also prove Eğecioğlu, Redmond, and Ryavec's identity (6) and the related identity

$$
\operatorname{det}\left(s_{i+j-1}(u)\right)_{0 \leq i, j \leq n-1}=U_{n} / u, \quad n>0,
$$

where $s_{-1}(u)=u^{-1}$. When $u=1,(9)$ reduces to (1) and when $u=3$, (9) reduces to

$$
\operatorname{det}\left(r_{i+j-1}\right)_{0 \leq i, j \leq n-1}=U_{n} / 3, \quad n>0 .
$$

Note that $r_{n-1}=\frac{1}{3}\left(\begin{array}{c}3 n \\ n\end{array}\right)$, so (10) is equivalent to $\operatorname{det}\left(\left(\begin{array}{c}3 n \\ n\end{array}\right)\right)_{0 \leq i, j \leq n-1}=3^{n-1} U_{n}$ for $n>0$. 
In Section 5 we consider the Hankel determinants of the coefficients of

$$
\frac{1-(1-9 x)^{1 / 3}}{3 x} \text {. }
$$

We first evaluate them using continued fractions, and then show that the method of Section 4 transforms them into powers of 3 times the determinant

$$
\operatorname{det}\left(\left(\begin{array}{l}
i+j \\
i-1
\end{array}\right)+\delta_{i j}\right)_{0 \leq i, j \leq n-1},
$$

which counts descending plane partitions and alternating sign matrices. Similarly, the Hankel determinant corresponding to

$$
\frac{1-(1-9 x)^{2 / 3}}{3 x}
$$

is transformed to a power of 3 times the determinant

$$
\operatorname{det}\left(\left(\begin{array}{c}
i+j \\
i
\end{array}\right)+\delta_{i j}\right)_{0 \leq i, j \leq n-1},
$$

which counts cyclically symmetric plane partitions.

Determinants of binomial coefficients can often be interpreted as counting configurations of non-intersecting paths (see, for example, Gessel and Viennot [11] and Bressoud [5]) and both sides of (7) (8) have such interpretations. In Section 6, we describe the nonintersecting lattice path interpretation for (7). We give a new class of interpretations of $a_{n}$ in terms of certain paths called $K$-paths in Theorem 6.3. From this new interpretation of $a_{n},(7)$ follows easily. The proof of Theorem 6.3 relies on a "sliding lemma", which says that the number of certain $K$-paths does not change after sliding their starting and ending points.

In Section 7, we study another class of paths called $T$-paths, which are related to trinomial coefficients, and $K T$-paths, which are analogous to $K$-paths. We find another class of interpretations of $a_{n}$ in terms of $K T$-paths, using which we find a new determinant identity involving $U_{n}$ (Theorem 7.3). Unfortunately, we do not have a nonintersecting path interpretation for this determinant. There is a natural bijection from $K$-paths to $K T$-paths, and the sliding lemma for $K T$-paths is easier to prove than that for $K$-paths.

In Section 8, we study $K T^{(r)}$-paths, which reduce to $K T$ paths when $r=2$. The results of Section 7 generalize, and we obtain determinant identities involving Hankel determinants for the number of $(r+1)$-ary trees (see $(72)$ and $(73))$.

In Section 9, we give algebraic proofs of the results of Section 8 using partial fractions.

\section{Hankel Determinants and Gauss's Continued Frac- tion}

Let $A(x)=\sum_{n \geq 0} A_{n} x^{n}$ be a formal power series. We define the Hankel determinants $H_{n}^{(k)}(A)$ of $A(x)$ by

$$
H_{n}^{(k)}(A)=\operatorname{det}\left(A_{i+j+k}\right)_{0 \leq i, j \leq n-1} .
$$


We shall write $H_{n}(A)$ for $H_{n}^{(0)}(A)$ and $H_{n}^{1}(A)$ for $H_{n}^{(1)}(A)$. We also define $\hat{H}_{n}(A)$ to be $H_{n}\left(A\left(x^{2}\right)\right)$. It is not difficult to show that $\hat{H}_{2 n}(A)=H_{n}(A) H_{n}^{1}(A)$ and $\hat{H}_{2 n+1}(A)=$ $H_{n+1}(A) H_{n}^{1}(A)$.

Let $g(x)$ be the generating function for ternary trees:

$$
g(x)=\sum_{n \geq 0} a_{n} x^{n}=\sum_{n \geq 0} \frac{1}{2 n+1}\left(\begin{array}{c}
3 n \\
n
\end{array}\right) x^{n},
$$

which is uniquely determined by the functional equation

$$
g(x)=1+x g(x)^{3} .
$$

Then $U_{n}=H_{n}(g(x)), V_{n}=H_{n}^{1}(g(x))$, and $W_{n}=\hat{H}_{n}(g(x))$.

In general, it is difficult to say much about $H_{n}(A(x))$. However, if $A(x)$ can be expressed as a continued fraction, then there is a very nice formula. This is the case for $g(x)$ : Tamm [28] observed that $g(x)$ has a nice continued fraction expression, which is a special case of Gauss's continued fraction. We introduce some notation to explain Tamm's approach.

We use the notation $S\left(x ; \lambda_{1}, \lambda_{2}, \lambda_{3}, \ldots\right)$ to denote the continued fraction

$$
S\left(x ; \lambda_{1}, \lambda_{2}, \lambda_{3}, \ldots\right)=\frac{1}{1-\frac{\lambda_{1} x}{1-\frac{\lambda_{2} x}{1-\frac{\lambda_{3} x}{\ddots}}}}
$$

The following theorem is equivalent to [14, Theorem 7.2]. Additional information about continued fractions and Hankel determinants can be found in Krattenthaler [17, Section $5.4]$.

Lemma 2.1. Let $A(x)=S\left(x ; \lambda_{1}, \lambda_{2}, \lambda_{3}, \ldots\right)$ and let $\mu_{i}=\lambda_{1} \lambda_{2} \cdots \lambda_{i}$. Then for $n \geq 1$,

$$
\begin{aligned}
& H_{n}(A)=\left(\lambda_{1} \lambda_{2}\right)^{n-1}\left(\lambda_{3} \lambda_{4}\right)^{n-2} \cdots\left(\lambda_{2 n-3} \lambda_{2 n-2}\right)=\mu_{2} \mu_{4} \cdots \mu_{2 n-2} \\
& H_{n}^{1}(A)=\lambda_{1}^{n}\left(\lambda_{2} \lambda_{3}\right)^{n-1} \cdots\left(\lambda_{2 n-2} \lambda_{2 n-1}\right)=\mu_{1} \mu_{3} \cdots \mu_{2 n-1} \\
& \hat{H}_{n}(A)=\lambda_{1}^{n-1} \lambda_{2}^{n-2} \cdots \lambda_{n-2}^{2} \lambda_{n-1}=\mu_{1} \mu_{2} \cdots \mu_{n-1} .
\end{aligned}
$$

We define the hypergeometric series by

$$
{ }_{2} F_{1}(a, b ; c \mid x)=\sum_{n=0}^{\infty} \frac{(a)_{n}(b)_{n}}{n !(c)_{n}} x^{n},
$$

where $(u)_{n}=u(u+1) \cdots(u+n-1)$.

Gauss proved the following theorem [14, Theorem 6.1], which gives a continued fraction for a quotient of two hypergeometric series: 
Lemma 2.2. If $c$ is not a negative integer then we have the continued fraction

$$
{ }_{2} F_{1}(a, b+1 ; c+1 \mid x) /{ }_{2} F_{1}(a, b ; c \mid x)=S\left(x ; \lambda_{1}, \lambda_{2}, \ldots\right),
$$

where

$$
\begin{aligned}
\lambda_{2 n-1} & =\frac{(a+n-1)(c-b+n-1)}{(c+2 n-2)(c+2 n-1)}, & & n=1,2, \ldots, \\
\lambda_{2 n} & =\frac{(b+n)(c-a+n)}{(c+2 n-1)(c+2 n)}, & n & =1,2, \ldots
\end{aligned}
$$

Combining Lemmas 2.1 and 2.2 gives a formula for evaluating certain Hankel determinants.

Lemma 2.3. Let

$$
A(x)={ }_{2} F_{1}(a, b+1 ; c+1 \mid \rho x) /{ }_{2} F_{1}(a, b ; c \mid \rho x) .
$$

Then

$$
\begin{aligned}
H_{n}(A) & =\prod_{i=0}^{n-1} \frac{(a)_{i}(b+1)_{i}(c-b)_{i}(c-a+1)_{i}}{(c)_{2 i}(c+1)_{2 i}} \rho^{2 i} \\
H_{n}^{1}(A) & =\prod_{i=1}^{n} \frac{(a)_{i}(b+1)_{i-1}(c-b)_{i}(c-a+1)_{i-1}}{(c)_{2 i-1}(c+1)_{2 i-1}} \rho^{2 i-1} \\
& =\prod_{i=1}^{n} \frac{(c-1) c}{b(c-a) \rho} \frac{(a)_{i}(b)_{i}(c-b)_{i}(c-a)_{i}}{(c)_{2 i}(c-1)_{2 i}} \rho^{2 i}
\end{aligned}
$$

Proof. By Lemma 2.2, $A(x)$ has the continued fraction expansion $A(x)=S\left(x ; \lambda_{1}, \lambda_{2}, \cdots\right)$ where

$$
\begin{aligned}
\lambda_{2 n-1} & =\frac{(a+n-1)(c-b+n-1)}{(c+2 n-2)(c+2 n-1)} \rho, \\
\lambda_{2 n} & =\frac{(b+n)(c-a+n)}{(c+2 n-1)(c+2 n)} \rho .
\end{aligned}
$$

Then

$$
\lambda_{1} \lambda_{3} \cdots \lambda_{2 i-1}=\frac{(a)_{i}(c-b)_{i}}{(c)_{2 i}} \rho^{i}
$$

and

$$
\lambda_{2} \lambda_{4} \cdots \lambda_{2 i}=\frac{(b+1)_{i}(c-a+1)_{i}}{(c+1)_{2 i}} \rho^{i} .
$$

So with the notation of Lemma 2.1,

$$
\mu_{2 i}=\lambda_{1} \lambda_{2} \cdots \lambda_{2 i}=\frac{(a)_{i}(c-b)_{i}(b+1)_{i}(c-a+1)_{i}}{(c)_{2 i}(c+1)_{2 i}} \rho^{2 i}
$$


and

$$
\mu_{2 i-1}=\lambda_{1} \lambda_{2} \cdots \lambda_{2 i-1}=\frac{(a)_{i}(c-b)_{i}(b+1)_{i-1}(c-a+1)_{i-1}}{(c)_{2 i}(c+1)_{2 i-2}} \rho^{2 i-1} .
$$

Then (19) follows immediately from (14), and (20) follows from (15) with the help of the identity $(c)_{2 i}(c+1)_{2 i-2}=(c)_{2 i-1}(c+1)_{2 i-1}$, and (21) follows easily from 20 .

There is also a simple formula for $H_{n}^{(2)}(A)$, although we will not need it.

Lemma 2.4. Let $Q(a, b, c \mid x)={ }_{2} F_{1}(a, b+1 ; c+1 \mid x) /{ }_{2} F_{1}(a, b ; c \mid x)$. Then

$$
Q(b, a, c \mid x)=\frac{c(a-b)}{a(c-b)}+\frac{b(c-a)}{a(c-b)} Q(a, b, c \mid x) .
$$

Proof. The formula is an immediate consequence of the contiguous relation $c(a-b)_{2} F_{1}(a, b ; c \mid x)+b(c-a){ }_{2} F_{1}(a, b+1 ; c+1 \mid x)+a(b-c)_{2} F_{1}(a+1, b ; c+1 \mid x)=0$, which is easily proved by equating coefficients of powers of $x$.

Equivalently, Lemma 2.4 asserts that $c a+b(c-a) Q(a, b, c \mid x)$ is symmetric in $a$ and $b$.

Proposition 2.5. With $A(x)$ as in Lemma 2.3, we have

$$
H_{n}^{(2)}(A)=\left(\frac{a(c-b)}{c(a-b)} \frac{(a+1)_{n}(c-b+1)_{n}}{(b+1)_{n}(c-a+1)_{n}}-\frac{b(c-a)}{c(a-b)}\right) H_{n+1}(A) .
$$

Proof. First note that if $u(x)=\alpha+\beta v(x)$, where $\alpha$ and $\beta$ are constants, then

$$
H_{n+1}(u)=\beta^{n+1} H_{n+1}(v)+\alpha \beta^{n} H_{n}^{(2)}(v),
$$

SO

$$
H_{n}^{(2)}(v)=\frac{1}{\alpha \beta^{n}} H_{n+1}(u)-\frac{\beta}{\alpha} H_{n+1}(v) .
$$

Now take $u=Q(b, a, c \mid x)$ and $v=Q(a, b, c \mid x)$, so that $u=\alpha+\beta v$ by Lemma 2.4, where $\alpha=c(a-b) / a(c-b)$ and $\beta=b(c-a) / a(c-b)$. Then by Lemma 2.3, we have

$$
\begin{aligned}
\frac{H_{n+1}(u)}{H_{n+1}(v)} & =\prod_{i=1}^{n} \frac{(a+1)_{i}}{(a)_{i}} \frac{(b)_{i}}{(b+1)_{i}} \frac{(c-b+1)_{i}}{(c-b)_{i}} \frac{(c-a)_{i}}{(c-a+1)_{i}} \\
& =\prod_{i=1}^{n} \frac{b(c-a)}{a(c-b)} \frac{(a+i)(c-b+i)}{(b+i)(c-a+i)}=\left[\frac{b(c-a)}{a(c-b)}\right]^{n} \frac{(a+1)_{n}(c-b+1)_{n}}{(b+1)_{n}(c-a+1)_{n}}
\end{aligned}
$$

and by (22) we have

$$
\frac{H_{n}^{(2)}(v)}{H_{n+1}(v)}=\frac{a(c-b)}{c(a-b)}\left[\frac{a(c-b)}{b(c-a)}\right]^{n} \frac{H_{n+1}(u)}{H_{n+1}(v)}-\frac{b(c-a)}{c(a-b)} .
$$

The result follows from (23) and (24). 
Tamm [28] evaluated the determinants $U_{n}$ and $V_{n}$ by first showing that

$$
\sum_{n=0}^{\infty} a_{n} x^{n}={ }_{2} F_{1}\left(\frac{2}{3}, \frac{4}{3} ; \frac{3}{2} \mid \frac{27}{4} x\right) /{ }_{2} F_{1}\left(\frac{2}{3}, \frac{1}{3} ; \frac{1}{2} \mid \frac{27}{4} x\right) .
$$

Given (25), it follows from Lemma 2.3 that

$$
U_{n}=\prod_{i=1}^{n-1} \frac{\left(\frac{2}{3}\right)_{i}\left(\frac{1}{6}\right)_{i}\left(\frac{4}{3}\right)_{i}\left(\frac{5}{6}\right)_{i}}{\left(\frac{1}{2}\right)_{2 i}\left(\frac{3}{2}\right)_{2 i}}\left(\frac{27}{4}\right)^{2 i}
$$

and

$$
V_{n}=\prod_{i=0}^{n} \frac{2}{3} \frac{\left(\frac{2}{3}\right)_{i}\left(\frac{1}{6}\right)_{i}\left(\frac{1}{3}\right)_{i}\left(-\frac{1}{6}\right)_{i}}{\left(\frac{1}{2}\right)_{2 i}\left(-\frac{1}{2}\right)_{2 i}}\left(\frac{27}{4}\right)^{2 i}
$$

So (4) and (5) will follow from

$$
\frac{\left(\frac{2}{3}\right)_{i}\left(\frac{1}{6}\right)_{i}\left(\frac{4}{3}\right)_{i}\left(\frac{5}{6}\right)_{i}}{\left(\frac{1}{2}\right)_{2 i}\left(\frac{3}{2}\right)_{2 i}}\left(\frac{27}{4}\right)^{2 i}=\frac{(3 i+1)(6 i) !(2 i) !}{(4 i+1) !(4 i) !}
$$

and

$$
\frac{2}{3} \frac{\left(\frac{2}{3}\right)_{i}\left(\frac{1}{6}\right)_{i}\left(\frac{1}{3}\right)_{i}\left(-\frac{1}{6}\right)_{i}}{\left(\frac{1}{2}\right)_{2 i}\left(-\frac{1}{2}\right)_{2 i}}\left(\frac{27}{4}\right)^{2 i}=\frac{\left(\begin{array}{c}
6 i-2 \\
2 i
\end{array}\right)}{2\left(\begin{array}{c}
4 i-1 \\
2 i
\end{array}\right)}
$$

for $i \geq 1$. These identities are most easily verified by using the fact that if $A_{1}=B_{1}$ and $A_{i+1} / A_{i}=B_{i+1} / B_{i}$ for $i \geq 1$, then $A_{i}=B_{i}$ for all $i \geq 1$. It is interesting to note that although (26) holds for $i=0$, (27) does not.

\section{Hypergeometric series evaluations}

Let $f=g-1=\sum_{n=1}^{\infty} a_{n} x^{n}=\sum_{n=1}^{\infty} \frac{1}{2 n+1}\left(\begin{array}{c}3 n \\ n\end{array}\right) x^{n}$. In this section we study cases of Gauss's continued fraction (17) that can be expressed in terms of $f$. We found empirically that there are ten cases of (17) that can be expressed as polynomials in $f$. We believe there are no others, but we do not have a proof of this. Since $a \neq b$ in all of these cases, by Lemma 2.4 they must come in pairs which are the same, except for their constant terms, up to a constant factor. It turns out that one element of each of these pairs factors as $(1+f)(1+r f)$, where $r$ is $0,1, \frac{1}{2},-\frac{1}{2}$, or $\frac{2}{5}$, while the other does not factor nicely. We have no explanation for this phenomenon.

Note that (28a) is the same as (25). 
Theorem 3.1. We have the following cases of Gauss's continued fraction:

$$
\begin{aligned}
1+f & ={ }_{2} F_{1}\left(\frac{2}{3}, \frac{4}{3} ; \frac{3}{2} \mid \frac{27}{4} x\right) /{ }_{2} F_{1}\left(\frac{2}{3}, \frac{1}{3} ; \frac{1}{2} \mid \frac{27}{4} x\right) \\
(1+f)^{2} & ={ }_{2} F_{1}\left(\frac{4}{3}, \frac{5}{3} ; \frac{5}{2} \mid \frac{27}{4} x\right) /{ }_{2} F_{1}\left(\frac{4}{3}, \frac{2}{3} ; \frac{3}{2} \mid \frac{27}{4} x\right) \\
(1+f)\left(1+\frac{1}{2} f\right) & ={ }_{2} F_{1}\left(\frac{5}{3}, \frac{7}{3} ; \frac{7}{2} \mid \frac{27}{4} x\right) /{ }_{2} F_{1}\left(\frac{5}{3}, \frac{4}{3} ; \frac{5}{2} \mid \frac{27}{4} x\right) \\
(1+f)\left(1-\frac{1}{2} f\right) & ={ }_{2} F_{1}\left(\frac{5}{3}, \frac{7}{3} ; \frac{5}{2} \mid \frac{27}{4} x\right) /{ }_{2} F_{1}\left(\frac{5}{3}, \frac{4}{3} ; \frac{3}{2} \mid \frac{27}{4} x\right) \\
(1+f)\left(1+\frac{2}{5} f\right) & ={ }_{2} F_{1}\left(\frac{2}{3}, \frac{4}{3} ; \frac{5}{2} \mid \frac{27}{4} x\right) /{ }_{2} F_{1}\left(\frac{2}{3}, \frac{1}{3} ; \frac{3}{2} \mid \frac{27}{4} x\right)
\end{aligned}
$$

Their companions are

$$
\begin{aligned}
& 1-\frac{1}{2} f={ }_{2} F_{1}\left(\frac{1}{3}, \frac{5}{3} ; \frac{3}{2} \mid \frac{27}{4} x\right) /{ }_{2} F_{1}\left(\frac{1}{3}, \frac{2}{3} ; \frac{1}{2} \mid \frac{27}{4} x\right) \\
& 1+\frac{1}{5} f+\frac{1}{10} f^{2}={ }_{2} F_{1}\left(\frac{2}{3}, \frac{7}{3} ; \frac{5}{2} \mid \frac{27}{4} x\right) /{ }_{2} F_{1}\left(\frac{2}{3}, \frac{4}{3} ; \frac{3}{2} \mid \frac{27}{4} x\right) \\
& 1+\frac{6}{7} f+\frac{2}{7} f^{2}={ }_{2} F_{1}\left(\frac{4}{3}, \frac{8}{3} ; \frac{7}{2} \mid \frac{27}{4} x\right) /{ }_{2} F_{1}\left(\frac{4}{3}, \frac{5}{3} ; \frac{5}{2} \mid \frac{27}{4} x\right) \\
& 1-\frac{2}{5} f+\frac{2}{5} f^{2}={ }_{2} F_{1}\left(\frac{4}{3}, \frac{8}{3} ; \frac{5}{2} \mid \frac{27}{4} x\right) /{ }_{2} F_{1}\left(\frac{4}{3}, \frac{5}{3} ; \frac{3}{2} \mid \frac{27}{4} x\right) \\
& 1+\frac{1}{2} f+\frac{1}{7} f^{2}={ }_{2} F_{1}\left(\frac{1}{3}, \frac{5}{3} ; \frac{5}{2} \mid \frac{27}{4} x\right) /{ }_{2} F_{1}\left(\frac{1}{3}, \frac{2}{3} ; \frac{3}{2} \mid \frac{27}{4} x\right)
\end{aligned}
$$

In order to prove Theorem 3.1, we need formulas for some rational functions of $f$ that are easily proved by Lagrange inversion.

Lemma 3.2. Let $f=\sum_{n=1}^{\infty} \frac{1}{2 n+1}\left(\begin{array}{c}3 n \\ n\end{array}\right) x^{n}$. Then $f$ satisfies the functional equation $f=$ $x(1+f)^{3}$ and

$$
\begin{aligned}
f^{k} & =\sum_{n=k}^{\infty} \frac{k}{n}\left(\begin{array}{c}
3 n \\
n-k
\end{array}\right) x^{n} \\
(1+f)^{k} & =\sum_{n=0}^{\infty} \frac{k}{3 n+k}\left(\begin{array}{c}
3 n+k \\
n
\end{array}\right) x^{n} \\
\frac{(1+f)^{k+1}}{1-2 f} & =\sum_{n=0}^{\infty}\left(\begin{array}{c}
3 n+k \\
n
\end{array}\right) x^{n} .
\end{aligned}
$$


In particular,

$$
\begin{aligned}
1+f & ={ }_{2} F_{1}\left(\frac{1}{3}, \frac{2}{3} ; \frac{3}{2} \mid \frac{27}{4} x\right) \\
\frac{1+f}{1-2 f} & ={ }_{2} F_{1}\left(\frac{1}{3}, \frac{2}{3} ; \frac{1}{2} \mid \frac{27}{4} x\right) \\
\frac{(1+f)^{2}}{1-2 f} & ={ }_{2} F_{1}\left(\frac{4}{3}, \frac{2}{3} ; \frac{3}{2} \mid \frac{27}{4} x\right) .
\end{aligned}
$$

Proof. We use the following form of the Lagrange inversion formula (see [9, Theorem 2.1] or [13, Theorem 1.2.4]): If $G(t)$ is a formal power series, then there is a unique formal power series $h=h(x)$ satisfying $h=x G(h)$, and

$$
\begin{aligned}
{\left[x^{n}\right] h^{k} } & =\frac{k}{n}\left[t^{n-k}\right] G(t)^{n}, \text { for } n, k>0, \\
{\left[x^{n}\right] \frac{h^{k}}{1-x G^{\prime}(h)} } & =\left[t^{n-k}\right] G(t)^{n}, \text { for } n, k \geq 0 .
\end{aligned}
$$

Let us define $f$ to be the unique formal power series satisfying $f=x(1+f)^{3}$. With $G(t)=(1+t)^{3},(36)$ gives $(30)$, and the case $k=1$ gives that the coefficient of $x^{n}$ in $f$ for $n \geq 1$ is $\frac{1}{n}\left(\begin{array}{c}3 n \\ n-1\end{array}\right)=\frac{1}{2 n+1}\left(\begin{array}{c}3 n \\ n\end{array}\right)$.

Replacing with $f$ with $x(1+f)^{3}$ and $k$ with $j$ in (30), and dividing both sides by $x^{j}$, gives

$$
(1+f)^{3 j}=\sum_{n=0}^{\infty} \frac{j}{n+j}\left(\begin{array}{c}
3 n+3 j \\
n
\end{array}\right) x^{n} .
$$

Since the coefficient of $x^{n}$ on each side is a polynomial in $j$, we may set $j=k / 3$ to obtain (31).

From (37) we have

$$
\frac{f^{j}}{1-3 x(1+f)^{2}}=\sum_{n=j}^{\infty}\left(\begin{array}{c}
3 n \\
n-j
\end{array}\right) x^{n} .
$$

Replacing $f$ by $x(1+f)^{3}$ in the numerator, and replacing $x(1+f)^{2}$ by $f /(1+f)$ in the denominator, gives

$$
\begin{aligned}
\frac{x^{j}(1+f)^{3 j+1}}{1-2 f} & =\sum_{n=j}^{\infty}\left(\begin{array}{c}
3 n \\
n-j
\end{array}\right) x^{n} \\
& =\sum_{n=0}^{\infty}\left(\begin{array}{c}
3 n+3 j \\
n
\end{array}\right) x^{n+j},
\end{aligned}
$$

SO

$$
\frac{(1+f)^{3 j+1}}{1-2 f}=\sum_{n=0}^{\infty}\left(\begin{array}{c}
3 n+3 j \\
n
\end{array}\right) x^{n} .
$$

As before, we may set $j=k / 3$ to obtain (32). 
Proof of Theorem 3.1. Formulas (28a)-(28e) follow from the evaluations of their numerators and denominators: (33), (34), (35), and

$$
\begin{aligned}
& { }_{2} F_{1}\left(\frac{2}{3}, \frac{4}{3} ; \frac{5}{2} \mid \frac{27}{4} x\right)=(1+f)^{2}\left(1+\frac{2}{5} f\right) \\
& { }_{2} F_{1}\left(\frac{4}{3}, \frac{5}{3} ; \frac{5}{2} \mid \frac{27}{4} x\right)=\frac{(1+f)^{4}}{1-2 f} \\
& { }_{2} F_{1}\left(\frac{5}{3}, \frac{7}{3} ; \frac{7}{2} \mid \frac{27}{4} x\right)=\frac{(1+f)^{5}\left(1+\frac{1}{2} f\right)}{1-2 f} \\
& { }_{2} F_{1}\left(\frac{4}{3}, \frac{5}{3} ; \frac{3}{2} \mid \frac{27}{4} x\right)=\frac{(1+f)^{4}}{(1-2 f)^{3}} \\
& { }_{2} F_{1}\left(\frac{5}{3}, \frac{7}{3} ; \frac{5}{2} \mid \frac{27}{4} x\right)=\frac{(1+f)^{5}\left(1-\frac{1}{2} f\right)}{(1-2 f)^{3}} .
\end{aligned}
$$

Our original derivations of these formulas were through the ${ }_{2} F_{1}$ contiguous relations $[1$, p. 558], but once we have found them, we can verify (38)-(40) by by taking appropriate linear combinations of (30) and (32). Formulas (41) and (42) can be proved by applying the formula

$$
{ }_{2} F_{1}(a+1, b+1 ; c+1 \mid x)=\frac{c}{a b} \frac{d}{d x}{ }_{2} F_{1}(a, b ; c \mid x)
$$

to (34) and (35) and using the fact that $d f / d x=(1+f)^{4} /(1-2 f)$.

Formulas (29a)-(29e) can be proved similarly; alternatively, they can be derived from (28a)-(28e) by using Lemma 2.4 .

Now we apply Lemma 2.3 to the formulas of Theorem 3.1. First we normalize the coefficient sequences that occur in (28a)-(28e) to make them integers, using (30) to find formulas for the coefficients. We define the sequence $a_{n}, b_{n}, c_{n}, d_{n}$, and $e_{n}$ by

$$
\begin{aligned}
1+f & =\sum_{n=0}^{\infty} a_{n} x^{n} & a_{n} & =\frac{(3 n) !}{n !(2 n+1) !}=\frac{1}{2 n+1}\left(\begin{array}{c}
3 n \\
n
\end{array}\right) \\
(1+f)^{2} & =\sum_{n=0}^{\infty} b_{n} x^{n} & b_{n} & =\frac{(3 n+1) !}{(n+1) !(2 n+1) !}=\frac{1}{n+1}\left(\begin{array}{c}
3 n+1 \\
n
\end{array}\right) \\
(1+f)(2+f) & =\sum_{n=0}^{\infty} c_{n} x^{n} & c_{n} & =2 \frac{(3 n) !}{(n+1) !(2 n) !}=\frac{2}{n+1}\left(\begin{array}{c}
3 n \\
n
\end{array}\right)=a_{n}+b_{n} \\
(1+f)(2-f) & =\sum_{n=0}^{\infty} d_{n} x^{n} & d_{n} & =2 \frac{(3 n) !}{(n+1) !(2 n+1) !}=3 a_{n}-b_{n} \\
(1+f)(5+2 f) & =\sum_{n=0}^{\infty} e_{n} x^{n} & e_{n} & =(9 n+5) \frac{(3 n) !}{(n+1) !(2 n+1) !}=3 a_{n}+2 b_{n}
\end{aligned}
$$

Here is a table of the first few values of these numbers 


\begin{tabular}{r|rrrrrrrr}
$n$ & 0 & 1 & 2 & 3 & \multicolumn{1}{c}{4} & \multicolumn{1}{c}{5} & \multicolumn{1}{c}{6} & \multicolumn{1}{c}{7} \\
\hline$a_{n}$ & 1 & 1 & 3 & 12 & 55 & 273 & 1428 & 7752 \\
$b_{n}$ & 1 & 2 & 7 & 30 & 143 & 728 & 3876 & 21318 \\
$c_{n}$ & 2 & 3 & 10 & 42 & 198 & 1001 & 5304 & 29070 \\
$d_{n}$ & 2 & 1 & 2 & 6 & 22 & 91 & 408 & 1938 \\
$e_{n}$ & 5 & 7 & 23 & 96 & 451 & 2275 & 12036 & 65892
\end{tabular}

The sequences $a_{n}$ and $b_{n}$ are well-known, and have simple combinatorial interpretations in terms of lattice paths: $a_{n}$ is the number of paths, with steps $(1,0)$ and $(0,1)$, from $(0,0)$ to $(2 n, n)$ that never rise above (but may touch) the line $x=2 y$ and $b_{n}$ is the number of paths from $(0,0)$ to $(2 n, n)$ that never rise above (but may touch) the line $x=2 y-1$ (see, e.g., Gessel [10]). Moreover, for $n>0, d_{n}$ is the number of two-stack-sortable permutations of $\{1,2, \ldots, n\}$. (See, e.g., West [29] and Zeilberger [30].) The sequences $c_{n}$ and $e_{n}$ are apparently not well-known.

Let us write $H_{n}(a)$ for $H_{n}\left(\sum_{n=0}^{\infty} a_{n} x^{n}\right)$, and similarly for other letters replacing $a$. Then applying Lemma 2.3 and Theorem 3.1 gives

$$
\begin{aligned}
& H_{n}(a)=\prod_{i=0}^{n-1} \frac{\left(\frac{2}{3}\right)_{i}\left(\frac{1}{6}\right)_{i}\left(\frac{4}{3}\right)_{i}\left(\frac{5}{6}\right)_{i}}{\left(\frac{1}{2}\right)_{2 i}\left(\frac{3}{2}\right)_{2 i}}\left(\frac{27}{4}\right)^{2 i} \\
& H_{n}^{1}(a)=\prod_{i=1}^{n} \frac{2}{3} \frac{\left(\frac{2}{3}\right)_{i}\left(\frac{1}{6}\right)_{i}\left(\frac{1}{3}\right)_{i}\left(-\frac{1}{6}\right)_{i}}{\left(\frac{1}{2}\right)_{2 i}\left(-\frac{1}{2}\right)_{2 i}}\left(\frac{27}{4}\right)^{2 i} \\
& H_{n}(b)=\prod_{i=0}^{n-1} \frac{\left(\frac{4}{3}\right)_{i}\left(\frac{5}{6}\right)_{i}\left(\frac{5}{3}\right)_{i}\left(\frac{7}{6}\right)_{i}}{\left(\frac{3}{2}\right)_{2 i}\left(\frac{5}{2}\right)_{2 i}}\left(\frac{27}{4}\right)^{2 i} \\
& H_{n}^{1}(b)=\prod_{i=1}^{n} \frac{\left(\frac{4}{3}\right)_{i}\left(\frac{5}{6}\right)_{i}\left(\frac{2}{3}\right)_{i}\left(\frac{1}{6}\right)_{i}}{\left(\frac{3}{2}\right)_{2 i}\left(\frac{1}{2}\right)_{2 i}}\left(\frac{27}{4}\right)^{2 i} \\
& H_{n}(c)=\prod_{i=0}^{n-1} 2 \frac{\left(\frac{5}{3}\right)_{i}\left(\frac{7}{6}\right)_{i}\left(\frac{7}{3}\right)_{i}\left(\frac{11}{6}\right)_{i}}{\left(\frac{5}{2}\right)_{2 i}\left(\frac{7}{2}\right)_{2 i}}\left(\frac{27}{4}\right)^{2 i} \\
& H_{n}^{1}(c)=\prod_{i=1}^{n} \frac{\left(\frac{5}{3}\right)_{i}\left(\frac{7}{6}\right)_{i}\left(\frac{4}{3}\right)_{i}\left(\frac{5}{6}\right)_{i}}{\left(\frac{5}{2}\right)_{2 i}\left(\frac{3}{2}\right)_{2 i}}\left(\frac{27}{4}\right)^{2 i} \\
& H_{n}(d)=\prod_{i=0}^{n-1} 2 \frac{\left(\frac{5}{3}\right)_{i}\left(\frac{1}{6}\right)_{i}\left(\frac{7}{3}\right)_{i}\left(\frac{5}{6}\right)_{i}}{\left(\frac{5}{2}\right)_{2 i}\left(\frac{3}{2}\right)_{2 i}}\left(\frac{27}{4}\right)^{2 i} \\
& H_{n}^{1}(d)=(-1)^{n} \prod_{i=1}^{n} \frac{\left(\frac{5}{3}\right)_{i}\left(\frac{1}{6}\right)_{i}\left(\frac{4}{3}\right)_{i}\left(-\frac{1}{6}\right)_{i}}{\left(\frac{3}{2}\right)_{2 i}\left(\frac{1}{2}\right)_{2 i}}\left(\frac{27}{4}\right)^{2 i}
\end{aligned}
$$




$$
\begin{aligned}
& H_{n}(e)=\prod_{i=0}^{n-1} 5 \frac{\left(\frac{2}{3}\right)_{i}\left(\frac{7}{6}\right)_{i}\left(\frac{4}{3}\right)_{i}\left(\frac{11}{6}\right)_{i}}{\left(\frac{3}{2}\right)_{2 i}\left(\frac{5}{2}\right)_{2 i}}\left(\frac{27}{4}\right)^{2 i} \\
& H_{n}^{1}(e)=\prod_{i=1}^{n} 2 \frac{\left(\frac{2}{3}\right)_{i}\left(\frac{7}{6}\right)_{i}\left(\frac{1}{3}\right)_{i}\left(\frac{5}{6}\right)_{i}}{\left(\frac{3}{2}\right)_{2 i}\left(\frac{1}{2}\right)_{2 i}}\left(\frac{27}{4}\right)^{2 i}
\end{aligned}
$$

Here is a table of the values of these Hankel determinants:

\begin{tabular}{r|rrrrrrr}
$n$ & 1 & 2 & 3 & 4 & 5 & \multicolumn{1}{c}{6} & \multicolumn{1}{c}{7} \\
\hline$H_{n}(a)$ & 1 & 2 & 11 & 170 & 7429 & 920460 & 323801820 \\
$H_{n}^{1}(a)$ & 1 & 3 & 26 & 646 & 45885 & 9304650 & 5382618660 \\
$H_{n}(b)$ & 1 & 3 & 26 & 646 & 45885 & 9304650 & 5382618660 \\
$H_{n}^{1}(b)$ & 2 & 11 & 170 & 7429 & 920460 & 323801820 & 323674802088 \\
$H_{n}(c)$ & 2 & 11 & 170 & 7429 & 920460 & 323801820 & 323674802088 \\
$H_{n}^{1}(c)$ & 3 & 26 & 646 & 45885 & 9304650 & 5382618660 & 8878734657276 \\
$H_{n}(d)$ & 2 & 3 & 10 & 85 & 1932 & 120060 & 20648232 \\
$H_{n}^{1}(d)$ & 1 & 2 & 10 & 133 & 4830 & 485460 & 136112196 \\
$H_{n}(e)$ & 5 & 66 & 2431 & 252586 & 74327145 & 62062015500 & 147198472495020 \\
$H_{n}^{1}(e)$ & 7 & 143 & 8398 & 1411510 & 677688675 & 928501718850 & 3628173844041420
\end{tabular}

It is apparent from the table that

$$
U_{n}=H_{n}(a)=H_{n-1}^{1}(b)=H_{n-1}(c),
$$

and that $V_{n}=H_{n}^{1}(a)=H_{n}(b)=H_{n-1}^{1}(c)$, and these are easily verified from the formulas. The combinatorial interpretations of $U_{n}$ and $V_{n}$ have already been discussed. The numbers $H_{n}(e)$ were shown by Kuperberg [19, Theorem 5] to count certain alternating sign matrices. In Kuperberg's notation, $H_{n}(e)=A_{\mathrm{UU}}^{(2)}(4 n ; 1,1,1)$.

There are also Hankel determinant evaluations corresponding to (29a)-(29e), normalized to make the entries integers. These evaluations can be found in Krattenthaler [17, Theorem 30].

\section{Determinants and Two-Variable Generating Func- tions}

In this section we describe a method for transforming determinants whose entries are given as coefficients of generating functions. (A related approach was used in [8] to evaluate Hankel determinants of Bell numbers.) Using this technique, we are able to convert the determinants for $U_{n}$ and $V_{n}$ in (1) and (2) into the known determinant evaluations given in (7) and (8). (Conversely, the evaluations of these Hankel determinants give new proofs of (7) and (8).) These two determinants are special cases of a determinant evaluation of 
Mills, Robbins, and Rumsey [22] (see [16, Theorem 37] for related determinants):

$$
\begin{aligned}
& \operatorname{det}\left(\left(\begin{array}{c}
i+j+r \\
2 i-j
\end{array}\right)\right)_{0 \leq i, j \leq n-1} \\
& \quad=(-1)^{\chi(n \equiv 3 \bmod 4)} 2^{\left(\begin{array}{c}
n-1 \\
2
\end{array}\right)} \prod_{i=1}^{n-1} \frac{(r+i+1)_{\lfloor(i+1) / 2\rfloor}\left(-r-3 n+i+\frac{3}{2}\right)_{\lfloor i / 2\rfloor}}{(i)_{i}},
\end{aligned}
$$

where $\chi(S)=1$ if $S$ is true and $\chi(S)=0$ otherwise. There exist short direct proofs of (44) (see $[3,15,24])$, but no really simple proof.

Suppose that we have a two-variable generating function

$$
D(x, y)=\sum_{i, j=0}^{\infty} d_{i, j} x^{i} y^{j} .
$$

Let $[D(x, y)]_{n}$ be the determinant of the $n \times n$ matrix

$$
\left(d_{i, j}\right)_{0 \leq i, j \leq n-1} .
$$

The following rules can be used to transform the determinant $[D(x, y)]_{n}$ to a determinant with the same value:

Constant Rules. Let $c$ be a non-zero constant. Then

$$
[c D(x, y)]_{n}=c^{n}[D(x, y)]_{n},
$$

and

$$
[D(c x, y)]_{n}=c^{\left(\begin{array}{l}
n \\
2
\end{array}\right)}[D(x, y)]_{n} .
$$

Product Rule. If $u(x)$ is any formal power series with $u(0)=1$, then

$$
[u(x) D(x, y)]_{n}=[D(x, y)]_{n} .
$$

Composition Rule. If $v(x)$ is any formal power series with $v(0)=0$ and $v^{\prime}(0)=1$, then

$$
[D(v(x), y)]_{n}=[D(x, y)]_{n} .
$$

The product and composition rules hold because the transformed determinants are obtained from the original determinants by elementary row operations. Equivalently, the new matrix is obtained by multiplying the old matrix on the left by a matrix with determinant 1 . Note that all of these transformations can be applied to $y$ as well as to $x$.

The Hankel determinants $H_{n}(A)$ and $H_{n}^{1}(A)$ of a formal power series $A(x)$ are given by

$$
\begin{aligned}
& H_{n}(A)=\left[\frac{x A(x)-y A(y)}{x-y}\right]_{n}, \\
& H_{n}^{1}(A)=\left[\frac{A(x)-A(y)}{x-y}\right]_{n} .
\end{aligned}
$$


Proof of (7) and (8). The generating function for the Hankel determinant $H_{n}(g)$ is

$$
\frac{x g(x)-y g(y)}{x-y} .
$$

Since $f /(1+f)^{3}=x, f$ is the compositional inverse of $x /(1+x)^{3}$, and thus $f\left(x /(1+x)^{3}\right)=$ $x$. Since $g=1+f$, we have $g\left(x /(1+x)^{3}\right)=1+x$.

Now let us substitute $x \rightarrow x /(1+x)^{3}, y \rightarrow y /(1+y)^{3}$ in (47). After simplifying, we obtain

$$
\frac{(1-x y)(1+x)(1+y)}{1-x y^{2}-3 x y-x^{2} y} .
$$

Then dividing by $(1+x)(1+y)$, we get

$$
\frac{1-x y}{1-x y^{2}-3 x y-x^{2} y} \text {. }
$$

Next, we show that

$$
\frac{1-x y}{1-x y^{2}-3 x y-x^{2} y}=\sum_{i, j}\left(\begin{array}{c}
i+j \\
2 i-j
\end{array}\right) x^{i} y^{j} .
$$

Multiplying both sides of (48) by $1-x y^{2}-3 x y-x^{2} y$ and equating coefficients of $x^{m} y^{n}$ shows that (48) is equivalent to the recurrence

$$
\left(\begin{array}{c}
m+n \\
2 m-n
\end{array}\right)-\left(\begin{array}{c}
m+n-3 \\
2 m-n
\end{array}\right)-3\left(\begin{array}{c}
m+n-2 \\
2 m-n-1
\end{array}\right)-\left(\begin{array}{c}
m+n-3 \\
2 m-n-3
\end{array}\right)=\left\{\begin{array}{cl}
1, & \text { if } m=n=0 \\
-1, & \text { if } m=n=1 \\
0, & \text { otherwise }
\end{array}\right.
$$

where we interpret the binomial coefficient $\left(\begin{array}{l}a \\ b\end{array}\right)$ as 0 if either $a$ or $b$ is negative, and the verification of the recurrence is straightforward. (We will give another proof of (48) in Example 9.2.) This completes the proof of (7).

For equation (8), we need to consider the generating function

$$
(g(x)-g(y)) /(x-y) \text {. }
$$

Making the same substitution as before gives

$$
\frac{(1+x)^{3}(1+y)^{3}}{1-x y^{2}-3 x y-x^{2} y} .
$$

Dividing by $(1+x)^{2}(1+y)^{3}$ gives

$$
\frac{1+x}{1-x y^{2}-3 x y-x^{2} y}
$$

which can be shown, by the same method as before, to equal

$$
\sum_{i, j}\left(\begin{array}{c}
i+j+1 \\
2 i-j
\end{array}\right) x^{i} y^{j}
$$


Using the same approach, we can prove a result of Ĕgecioğlu, Redmond, and Ryavec [6] that gives another Hankel determinant for $V_{n}$. (It should be noted that our $V_{n}$ is their $V_{n-1}$.) In Section 4 of [6] they define numbers $\mu_{n}$ and gave several characterizations for them. In later sections they transform the Hankel determinant for these numbers several times, as described on page 5 of their paper, ultimately reducing it to the Hankel determinant for the numbers $\left(\begin{array}{c}3 n+2 \\ n\end{array}\right)$. We will give a direct reduction of the generating function for these Hankel determinants to (49).

In their Theorem 2, Ĕgecioğlu, Redmond, and Ryavec give several characterizations of the numbers $\mu_{n}$. We will use a characterization given not in the statement of this theorem, but in the proof, on page 16: the generating function

$$
M(x)=\sum_{n=0}^{\infty} \mu_{n} x^{n+1}
$$

satisfies

$$
M(x)=x+3 x M(x)^{2}+x M(x)^{3} .
$$

Theorem 4.1. Let $\mu_{n}$ be defined by (50) and (51). Then $\operatorname{det}\left(\mu_{i+j}\right)_{0 \leq i, j \leq n-1}=V_{n}$.

Proof. By (45),

$$
\operatorname{det}\left(\mu_{i+j}\right)_{0 \leq i, j \leq n-1}=\left[\frac{M(x)-M(y)}{x-y}\right]_{n} .
$$

By (51), $M(x)$ is the compositional inverse of $x /\left(1+3 x^{2}+x^{3}\right)$, so making the substitution $x \rightarrow x /\left(1+3 x^{2}+x^{3}\right), y \rightarrow y /\left(1+3 y^{2}+y^{3}\right)$ in $(M(x)-M(y)) /(x-y)$ and simplifying gives

$$
\frac{\left(1+3 x^{2}+x^{3}\right)\left(1+3 y^{2}+y^{3}\right)}{1-x y^{2}-3 x y-x^{2} y} .
$$

Applying the product rule, we reduce this generating function to (49), for which the corresponding determinant, as we have seen, is $V_{n}$.

We note that if (51) is replaced with $M(x)=x+\alpha x M(x)+3 x M(x)^{2}+x M(x)^{3}$, where $\alpha$ is arbitrary, then the Hankel determinants are unchanged.

To transform in this way the more general determinant on the left side of (44), we would start with the generating function

$$
\sum_{i, j}\left(\begin{array}{c}
i+j+r \\
2 i-j
\end{array}\right) x^{i} y^{j}=\frac{\sum_{n=0}^{\infty}\left[\left(\begin{array}{c}
r+n \\
2 n
\end{array}\right)+\left(\begin{array}{c}
r+n-2 \\
2 n-1
\end{array}\right) y\right] x^{n}}{1-x y^{2}-3 x y-x^{2} y} .
$$

The generating function in $r$ of (52) is derived in (77). The sums in the numerator can 
be evaluated explicitly by making an appropriate substitution in the identities

$$
\begin{aligned}
\sum_{n=0}^{\infty}\left(\begin{array}{c}
r+n \\
2 n
\end{array}\right)\left(-4 \sin ^{2} \theta\right)^{n} & =\frac{\cos (2 r+1) \theta}{\cos \theta} \\
\sum_{n=0}^{\infty}\left(\begin{array}{c}
r+n-2 \\
2 n-1
\end{array}\right)\left(-4 \sin ^{2} \theta\right)^{n} & =-2 \tan \theta \sin 2(r-1) \theta .
\end{aligned}
$$

However we have not been able to use these formulas to prove (44).

Another application of this method gives a family of generating functions that have the same Hankel determinants.

Theorem 4.2. Let $A(x)$ be a formal power series with $A(0)=1$ and let $c$ be a constant. Then we have

$$
H_{n}\left(\frac{A(x)}{1-c x A(x)}\right)=H_{n}(A)
$$

for all $n$, and

$$
H_{n}\left(\frac{1}{1-c x A(x)}\right)=c^{n-1} H_{n-1}^{1}(A)
$$

for $n \geq 1$.

Proof. We use the method of generating functions to evaluate these determinants. By $(45)$

$$
\begin{aligned}
& H_{n}\left(\frac{A(x)}{1-c x A(x)}\right)=\left[\frac{\frac{x A(x)}{1-c x A(x)}-\frac{y A(y)}{1-c y A(y)}}{x-y}\right]_{n} \\
& =\left[\frac{1}{(1-c x A(x))(1-c y A(y))} \frac{x A(x)-y A(y)}{x-y}\right]_{n} .
\end{aligned}
$$

Since $(1-c x A(x))^{-1}$ is a formal power series with constant term 1 , we get

$$
H_{n}\left(\frac{A(x)}{1-c x A(x)}\right)=\left[\frac{x A(x)-y A(y)}{x-y}\right]_{n}=H_{n}(A) .
$$

A similar computation shows that

$$
\begin{aligned}
H_{n}\left(\frac{1}{1-c x A(x)}\right) & =\left[1+c x y \frac{A(x)-A(y)}{x-y}\right]_{n} \\
& =\left[c \frac{A(x)-A(y)}{x-y}\right]_{n-1}=c^{n-1} H_{n-1}^{1}(A),
\end{aligned}
$$

since $[1+x y D(x, y)]_{n}$ is the determinant of a block matrix of two blocks, with the first block [1] and the second block $[D(x, y)]_{n-1}$. 
We now prove (6) and (9). First we set $c=u-1$ and $A=f / x$ in (53), getting

$$
V_{n}=\operatorname{det}\left(a_{i+j+1}\right)_{0 \leq i, j \leq n-1}=H_{n}(f / x)=H_{n}\left(\frac{f / x}{1+(1-u) f}\right) .
$$

Next we show that

$$
\frac{f / x}{1+(1-u) f}=\sum_{n=0}^{\infty} s_{n}(u) x^{n}
$$

where

$$
s_{n}(u)=\sum_{k=0}^{n} \frac{k+1}{n+1}\left(\begin{array}{c}
3 n-k+1 \\
n-k
\end{array}\right) u^{k} .
$$

We have

$$
\begin{aligned}
\frac{f / x}{1+(1-u) f} & =\frac{f / x}{1+f} \cdot \frac{1}{1-u f /(1+f)} \\
& =\frac{(1+f)^{2}}{1-u x(1+f)^{2}}, \text { since } f=x(1+f)^{3} \\
& =\sum_{k=0}^{\infty} u^{k} x^{k}(1+f)^{2 k+2} \\
& =\sum_{k=0}^{\infty} u^{k} x^{k} \sum_{m=0}^{\infty} \frac{2 k+2}{3 m+2 k+2}\left(\begin{array}{c}
3 m+2 k+2 \\
m
\end{array}\right) x^{m}, \text { by }(31), \\
& =\sum_{n=0}^{\infty} x^{n} \sum_{k=0}^{n} \frac{k+1}{n+1}\left(\begin{array}{c}
3 n-k+1 \\
n-k
\end{array}\right) u^{k},
\end{aligned}
$$

which proves (55). Then $s_{n}(1)=a_{n+1}$ from (55), $s_{n}(0)=\frac{1}{n+1}\left(\begin{array}{c}3 n+1 \\ n\end{array}\right)$ by setting $u=0$ in (56), and $s_{n}(3)=\left(\begin{array}{c}3 n+2 \\ n\end{array}\right)$ follows from (32). This completes the proof of $(6)$.

Next we prove (9), which by (55) is equivalent to

$$
H_{n}\left(u^{-1}+\frac{f}{1+(1-u) f}\right)=U_{n} / u .
$$

We have

$$
u^{-1}+\frac{f}{1+(1-u) f}=\frac{u^{-1}}{1-u x(1+f)^{2}},
$$

so by (54), the Hankel determinant is equal to $u^{-1} H_{n-1}^{1}\left((1+f)^{2}\right)$. In the notation of Section 3 , this is $u^{-1} H_{n-1}^{1}(b)$, which by (43) is equal to $u^{-1} U_{n}$.

We also have an analogue of Theorem 4.2 for the Hankel determinants $H_{n}^{1}$.

Theorem 4.3. Let $A(x)$ be a formal power series with $A(0)=1$ and let $c \neq 1$ be $a$ constant. Then we have

$$
H_{n}^{1}\left(\frac{A(x)}{1-c A(x)}\right)=(1-c)^{-2 n} H_{n}^{1}(A)
$$


Proof. We use the method of generating functions. By (46),

$$
\begin{aligned}
H_{n}^{1}\left(\frac{A(x)}{1-c A(x)}\right) & =\left[\frac{\frac{A(x)}{1-c A(x)}-\frac{A(y)}{1-c A(y)}}{x-y}\right]_{n} \\
& =\left[\frac{1}{(1-c A(x))(1-c A(y))} \frac{A(x)-A(y)}{x-y}\right]_{n} .
\end{aligned}
$$

Since $(1-c A(x))^{-1}$ is a formal power series with constant term $(1-c)^{-1}$ when $c \neq 1$, we get

$$
H_{n}^{1}\left(\frac{(1-c)^{2} A(x)}{1-c A(x)}\right)=\left[(1-c)^{-2} \frac{A(x)-A(y)}{x-y}\right]_{n}=(1-c)^{-2 n} H_{n}^{1}(A) .
$$

\section{A Hankel Determinant for the Number of Alter- nating Sign Matrices}

Let $\mathcal{A}_{n}$ be the number of $n \times n$ alternating sign matrices. It is well-known that

$$
\mathcal{A}_{n}=\prod_{k=0}^{n-1} \frac{(3 k+1) !}{(n+k) !}
$$

as conjectured by Mills Robbins and Rumsey [21] and proved by Zeilberger [31] and Kuperberg [18].

The numbers $\mathcal{A}_{n}$ also count totally symmetric, self-complementary plane partitions, as shown by Andrews [2]. We find, up to a power of 3, a Hankel determinant expression for $\mathcal{A}_{n}$.

Let

$$
\hat{C}(x)=\frac{1-(1-9 x)^{1 / 3}}{3 x} .
$$

The coefficients of $\hat{C}(x)$ are positive integers that are analogous to Catalan numbers. They have no known combinatorial interpretation and have been little studied, but they do appear in [20, Eq. 61].

Theorem 5.1. The number of $n \times n$ alternating sign matrices is

$$
\mathcal{A}_{n}=3^{-\left(\begin{array}{l}
n \\
2
\end{array}\right)} H_{n}(\hat{C}) .
$$

Proof. Let

$$
D(x, y)=(x \hat{C}(x)-y \hat{C}(y)) /(x-y)
$$

be the generating function for the Hankel determinant $H_{n}(\hat{C})$. It is easy to see that $D(x / \sqrt{3}, y / \sqrt{3})$ is the generating function for $3^{-\left(\begin{array}{c}n \\ 2\end{array}\right)} H_{n}(\hat{C})$. We make the substitution 
$x \rightarrow x-\sqrt{3} x^{2}+x^{3}, y \rightarrow y-\sqrt{3} y^{2}+y^{3}$ in $D(x / \sqrt{3}, y / \sqrt{3})$, and simplify. The generating function becomes

$$
\frac{1}{1-\sqrt{3}(x+y)+x^{2}+x y+y^{2}} \text {. }
$$

Let $\omega=-\frac{1}{2}-\frac{\sqrt{-3}}{2}$ be a cube root of unity. Make another substitution $x \rightarrow-\sqrt{-1} x /(1+$ $\omega x), y \rightarrow \sqrt{-1} y /\left(1+\omega^{2} y\right)$, and simplify. The generating function becomes

$$
\frac{(1+\omega x)^{2}\left(1+\omega^{2} y\right)^{2}}{(1-x y)(1-x-y)}
$$

Dividing by $(1+\omega x)^{2}\left(1+\omega^{2} y\right)^{2}$, the generating function becomes

$$
\frac{1}{(1-x-y)(1-x y)}
$$

Multiplying by $\left(1-x+x^{2}\right)(1-y) /(1-x)$, we get

$$
\frac{\left(1-x+x^{2}\right)(1-y)}{(1-x)(1-x-y)(1-x y)}=\frac{x}{y(1-x-y)}+\frac{1}{1-x y}-\frac{x}{y(1-x)} .
$$

Expanding the right-hand side of the above equation, we get

$$
H_{n}(\hat{C})=3^{\left(\begin{array}{c}
n \\
2
\end{array}\right)} \operatorname{det}\left(\left(\begin{array}{c}
i+j \\
i-1
\end{array}\right)+\delta_{i, j}\right)_{0 \leq i, j \leq n-1},
$$

where $\delta_{i, j}$ equals 1 if $i=j$ and 0 otherwise. The theorem then follows from a known formula for $\mathcal{A}_{n}[5$, p. 22].

Remark 5.2. We have another determinant expression

$$
\mathcal{A}_{n}=\operatorname{det}\left(\left(\begin{array}{c}
i+j \\
i
\end{array}\right)-\delta_{i, j+1}\right)
$$

since

$$
\frac{1}{(1-x-y)(1-x y)}=\frac{1}{1-y+y^{2}}\left(\frac{1}{1-x-y}-\frac{y}{1-x y}\right) .
$$

There is a result similar to Theorem 5.1

$$
\hat{C}_{1}(x)=\frac{1-(1-9 x)^{2 / 3}}{3 x} .
$$

Let $\mathcal{A}_{n}^{\prime}$ be the number of cyclically symmetric plane partitions in the $n$-cube. We have

\section{Theorem 5.3.}

$$
\mathcal{A}_{n}^{\prime}=3^{-\left(\begin{array}{l}
n \\
2
\end{array}\right)} H_{n}\left(\hat{C}_{1}\right)
$$


Proof. Let

$$
D(x, y)=\left(x \hat{C}_{1}(x)-y \hat{C}_{1}(y)\right) /(x-y)
$$

be the generating function for the Hankel determinant $H_{n}\left(\hat{C}_{1}\right)$. Similarly $D(x / \sqrt{3}, y / \sqrt{3})$ is the generating function for $3^{-\left(\begin{array}{c}n \\ 2\end{array}\right)} H_{n}\left(\hat{C}_{1}\right)$. We make the same substitution (as for $H_{n}(\hat{C})$ ) $x \rightarrow x-\sqrt{3} x^{2}+x^{3}, y \rightarrow y-\sqrt{3} y^{2}+y^{3}$, and simplify. The generating function becomes

$$
\frac{2-\sqrt{3}(x+y)}{1-\sqrt{3}(x+y)+x^{2}+x y+y^{2}} \text {. }
$$

Similarly, we make another substitution $x \rightarrow-\sqrt{-1} x /(1+\omega x), y \rightarrow \sqrt{-1} y /\left(1+\omega^{2} y\right)$, and simplify. The generating function becomes

$$
\frac{(2-x-y-x y)(1+\omega x)\left(1+\omega^{2} y\right)}{(1-x y)(1-x-y)} .
$$

Dividing by $(1+\omega x)\left(1+\omega^{2} y\right)$, the generating function becomes

$$
\frac{(2-x-y-x y)}{(1-x-y)(1-x y)}=\frac{1}{1-x-y}+\frac{1}{1-x y} \text {. }
$$

So we have

$$
H_{n}\left(\hat{C}_{1}\right)=3^{\left(\begin{array}{c}
n \\
2
\end{array}\right)} \operatorname{det}\left(\left(\begin{array}{c}
i+j \\
i
\end{array}\right)+\delta_{i, j}\right)_{0 \leq i, j \leq n-1},
$$

which is equal to $3^{\left(\begin{array}{c}n \\ 2\end{array}\right)} \mathcal{A}_{n}^{\prime}$. (See $[5$, p. $177,(5.28)]$.)

Since $\hat{C}(x)={ }_{2} F_{1}\left(\frac{2}{3}, 1 ; 2 \mid 9 x\right)$, we can find a continued fraction for $\hat{C}(x)$ by setting $a=\frac{2}{3}, b=0, c=1$ in Lemma 2.2, and thus evaluate the Hankel determinant for $\hat{C}(x)$ by Lemma 2.1. Similarly, since $\hat{C}_{1}(x)=2_{2} F_{1}\left(\frac{1}{3}, 1 ; 2 \mid 9 x\right)$, we can evaluate the Hankel determinant for $\hat{C}_{1}(x)$ be taking $a=\frac{1}{3}, b=0, c=1$ in Lemma 2.2.

The Hankel determinants $H_{n}(\hat{C})$ and $H_{n}\left(\hat{C}_{1}\right)$, can also be evaluated by applying a more general result (see, e.g., [16, Theorem 26, Eq. (3.12)]):

$$
\operatorname{det}\left(\left(\begin{array}{c}
A \\
L_{i}+j
\end{array}\right)\right)_{1 \leq i, j \leq n}=\frac{\prod_{1 \leq i<j \leq n}\left(L_{i}-L_{j}\right)}{\prod_{1 \leq i \leq n}\left(L_{i}+n\right) !} \frac{\prod_{1 \leq i \leq n}\left(L_{i}+A+1\right) !}{\prod_{1 \leq i \leq n}(A+1-i) !}
$$

where $L_{1}, \ldots, L_{n}$ and $A$ are indeterminates, and the factorials are interpreted using gamma functions when necessary.

Thus these calculations give a simple method of evaluating the determinants

$$
\operatorname{det}\left(\left(\begin{array}{c}
i+j \\
i-1
\end{array}\right)+\delta_{i, j}\right)_{0 \leq i, j \leq n-1} \text { and } \operatorname{det}\left(\left(\begin{array}{c}
i+j \\
i
\end{array}\right)+\delta_{i, j}\right)_{0 \leq i, j \leq n-1} .
$$

For more information on similar determinants, see Krattenthaler [16, Theorems 32-35] [17, Section 5.5]. 


\section{A Combinatorial Proof of (7)}

For the reader's convenience, we restate equation (7) as follows:

$$
\operatorname{det}\left(a_{i+j}\right)_{0 \leq i, j \leq n-1}=\operatorname{det}\left(\left(\begin{array}{c}
i+j \\
2 i-j
\end{array}\right)\right)_{0 \leq i, j \leq n-1} .
$$

Both sides of (63) have combinatorial meanings in terms of nonintersecting paths (see Gessel and Viennot [11]). The right-hand side counts $\mathcal{U}_{R}(n)$, the set of $n$-tuples of nonintersecting paths from $P_{0}^{\prime}, \ldots, P_{n-1}^{\prime}$ to $Q_{0}^{\prime}, \ldots, Q_{n-1}^{\prime}$, where $P_{i}^{\prime}=(i,-2 i)$ and $Q_{i}^{\prime}=(2 i,-i)$. For the paths to be nonintersecting, $P_{i}^{\prime}$ must go to $Q_{i}^{\prime}$. See the right picture of Figure 1. Mills, Robbins, and Rumsey [15] in fact gave a bijection from the type (a) objects of Section 1 to such $n$-tuples of lattice paths.
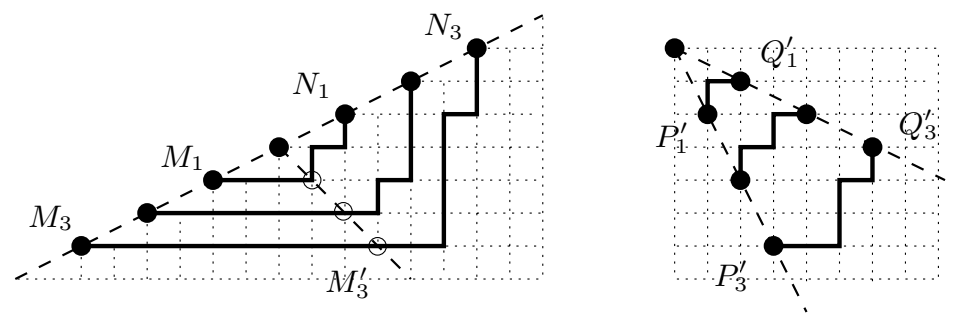

Figure 1: Lattice path interpretation of (63).

For the left-hand side, we notice that $a_{n}$ counts the number of paths from $(0,0)$ to $(2 n, n)$ that never go above the line $y=x / 2$. See, e.g., [10]. It is easy to see that the left-hand side of $(63)$ counts $\mathcal{U}_{L}(n)$, the set of $n$-tuples of nonintersecting paths that stay below the line $y=x / 2$, from $M_{0}, \ldots, M_{n-1}$ to $N_{0}, \ldots, N_{n-1}$, where $M_{i}=(-2 i,-i)$ and $N_{i}=(2 i, i)$. For the paths to be nonintersecting, $M_{i}$ must go to $N_{i}$. Moreover, from the left picture of Figure 1, we see that $M_{i}$ can be replaced with $M_{i}^{\prime}=(i,-i)$.

An interesting problem is to find a bijection from $\mathcal{U}_{L}(n)$ to $\mathcal{U}_{R}(n)$. Such a bijection will result in a combinatorial enumeration of the type $(a)$ objects.

Both $\mathcal{U}_{L}(n)$ and $\mathcal{U}_{R}(n)$ can be easily converted into variations of plane partitions. But we have not found it helpful.

We find an alternative bijective proof of (63). The algebraic idea behind the proof is the following matrix identity that implies (63):

$$
\begin{aligned}
&\left(a_{i+j}\right)_{0 \leq i, j \leq n-1}=\left(\frac{3 j+1}{3 i+1}\left(\begin{array}{c}
3 i+1 \\
i-j
\end{array}\right)\right)_{0 \leq i, j \leq n-1} \\
& \quad\left(\left(\begin{array}{c}
i+j \\
2 i-j
\end{array}\right)\right)_{0 \leq i, j \leq n-1}\left(\frac{3 i+1}{3 j+1}\left(\begin{array}{c}
3 j+1 \\
j-i
\end{array}\right)\right)_{0 \leq i, j \leq n-1},
\end{aligned}
$$

where

$$
\frac{3 i+1}{3 j+1}\left(\begin{array}{c}
3 j+1 \\
j-i
\end{array}\right)=\left[x^{i}\right] g f^{j}
$$


(See (30)). Note that the left (right) transformation matrix is a lower (upper) triangular matrix with diagonal entries 1 . The matrix identity is obtained by carefully analyzing the transformation we performed in Section 4 when proving (7).

The bijective proof relies on a new interpretation of $a_{n}$ in terms of certain paths that we call $K$-paths. The matrix identity (64) follows easily from the new interpretation. This gives a bijection from $\mathcal{U}_{R}(n)$ to $\mathcal{U}_{K}(n)$, the set of $n$-tuples of nonintersecting $K$ paths resulting from the new interpretation. The desired bijection could be completed by giving the bijection from $\mathcal{U}_{K}(n)$ to $\mathcal{U}_{L}(n)$. But we have not succeeded in this.

The new interpretation of $a_{n}$ consists of three kinds of paths: normal paths, $H_{2}$-paths, and $V_{2}$-paths. A normal path has steps $(0,1)$ and $(1,0)$. A path is an $H_{2}$ path if each horizontal step is $(2,0)$ instead of $(1,0)$. By dividing each horizontal 2-step into two horizontal 1-steps, we can represent an $H_{2}$ path as a normal path. Similarly, a path is a $V_{2}$ path if each vertical step is $(0,2)$.

By reflecting in the line $y=-x$, we can convert an $H_{2}$ path into a $V_{2}$ path, or a $V_{2}$ path into an $H_{2}$ path. This bijection can convert any property of $H_{2}$-paths into a similar property of $V_{2}$-paths.

It is well-known that the number of paths that start at $(0,0)$, end at $(n, 2 n)$, and never go above the line $y=2 x$ is $a_{n}=\frac{1}{2 n+1}\left(\begin{array}{c}3 n \\ n\end{array}\right)$. Replacing each horizontal step by two horizontal steps, it follows that:

Proposition 6.1. The number of $H_{2}$-paths (or $V_{2}$-paths) that start at $(0,0)$, end at $(2 n, 2 n)$ and never go above the diagonal equals $a_{n}$.

Definition 6.2. We call a path $P$ a K-path if it satisfies the following four conditions.

1. The path $P$ never goes above the diagonal.

2. The part of $P$ that is below the line $y=-2 x$ is a $V_{2}$ path.

3. The part of $P$ between the two lines $y=-2 x$ and $x=-2 y$ is a normal path.

4. The part of $P$ that is above the line $x=-2 y$ is an $\mathrm{H}_{2}$ path.

From the definition, we see that a $K$-path can be uniquely decomposed into three kinds of paths: a $V_{2}$ path, followed by a normal path, followed by an $H_{2}$ path. Depending on its starting point, some of the paths may be empty. The normal path region is between the two lines $y=-2 x$ and $x=-2 y$. The steps occurring in a $K$-path are shown in Figure 2. We have

Theorem 6.3. The number of $K$-paths from $(-2 m,-2 m)$ to $(2 n, 2 n)$, where $m+n \geq 0$, is $a_{m+n}$.

The proof of the theorem will be given later. From the new interpretation of $a_{n}$, $U_{n}$ counts $\mathcal{U}_{K}(n)$, the set of $n$-tuples of nonintersecting $K$-paths from $P_{0}, \ldots, P_{n-1}$ to $Q_{0}, \ldots, Q_{n-1}$, where $P_{i}=(-2 i,-2 i)$, and $Q_{i}=(2 i, 2 i)$ for $i=0,1, \ldots, n-1$. See Figure 2. For the paths to be nonintersecting, $P_{i}$ must go to $Q_{i}$. In such an $n$-tuples 


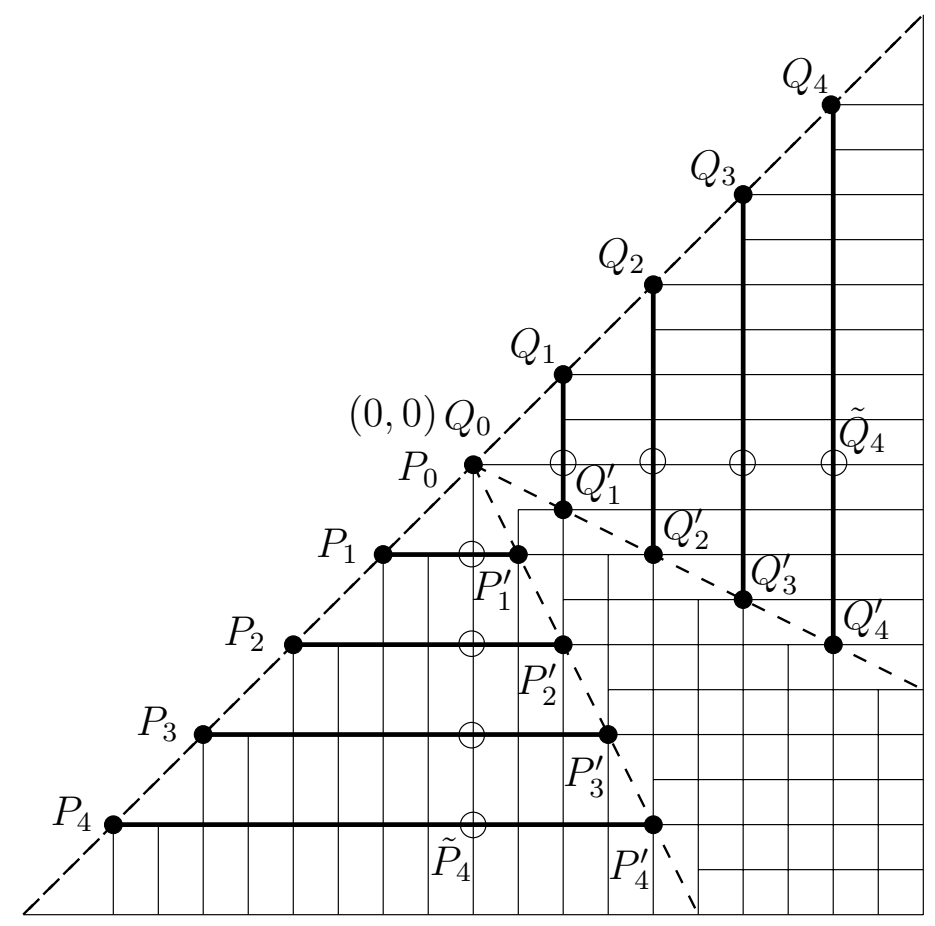

Figure 2: The grid for $K$-paths.

of nonintersecting $K$-paths, the path from $P_{i}$ to $Q_{i}$ must start with a path from $P_{i}=$ $(-2 i,-2 i)$ to $P_{i}^{\prime}=(i,-2 i)$, and end with a path from $Q_{i}^{\prime}=(2 i,-i)$ to $Q_{i}=(2 i, 2 i)$. So $\mathcal{U}_{K}(n)$ is in natural bijection with $\mathcal{U}_{R}(n)$. If we count the number of $K$-paths according to their intersections with the lines $y=-2 x$ and $x=-2 y$, we get the matrix identity (64).

If $P$ is a $K$-path from $(-2 m,-2 m)$ to $(2 n, 2 n)$ with $m \leq 0$ (or $n \leq 0)$, then $P$ is an $H_{2}$ (or a $V_{2}$ )-path, and Theorem 6.3 follows from Proposition 6.1. So we can assume that $m$ and $n$ are both positive integers.

The idea of the proof of Theorem 6.3 is to show that the number of $K$-paths from $(-2 m,-2 m)$ to $(2 n, 2 n)$ is unchanged after sliding their starting and ending points along the diagonal by $(2,2)$.

In fact, the following refinement is true. See Figure 3.

Lemma 6.4 (Sliding Lemma). The number of $K$-paths from $(i-2,-2 i-2)$ to $(2 j,-j)$ equals the number of $K$-paths from $(i,-2 i)$ to $(2 j+2,-j+2)$.

Proof. Let $N(i, j)$ be the number of $K$-paths from $A_{i}=(i-2,-2 i-2)$ to $B_{j}=(2 j,-j)$. It is clear that $N(i, j)=0$ if $i<0$ or $j<0$.

By reflecting in the line $y=-x$, we can give a bijective proof of the following statement: The number of $K$-paths from $(i,-2 i)$ to $(2 j+2,-j+2)$ equals the number of $K$-paths from $(j-2,-2 j-2)$ to $(2 i,-i)$, which is $N(j, i)$. Therefore it suffices to show that $N(i, j)=N(j, i)$. 


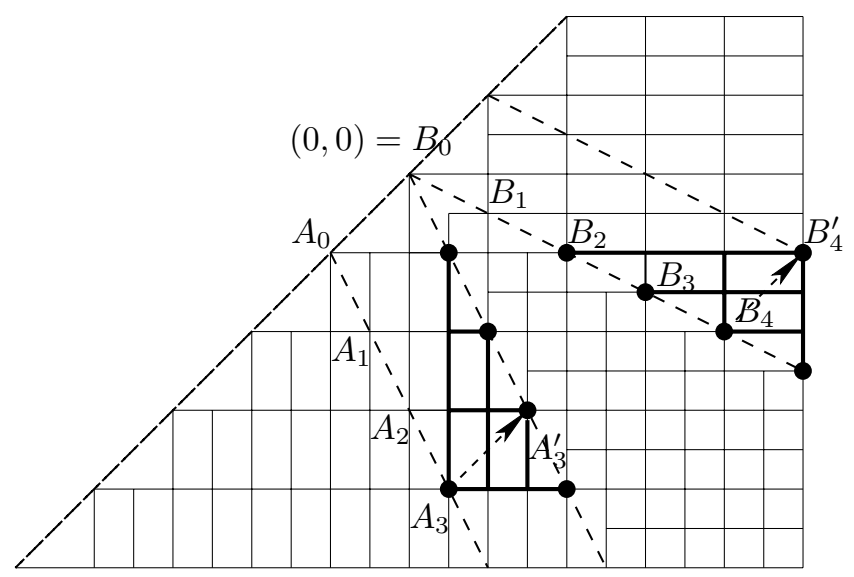

Figure 3: Picture for the sliding lemma.

The cases $i=0$ and $i=1$ correspond to starting at $A_{0}$ and $A_{1}$. From Figure 3, we can check that $N(i, j)=N(j, i)$ directly. We have

\begin{tabular}{c|ccccc}
$i \backslash j$ & 0 & 1 & 2 & 3 & 4 \\
\hline 0 & 1 & 2 & 1 & 0 & 0 \\
1 & 2 & 5 & 9 & 5 & 1 \\
2 & 1 & 9 & $*$ & $*$ & $*$ \\
3 & 0 & 5 & $*$ & $*$ & $*$ \\
4 & 0 & 1 & $*$ & $*$ & $*$
\end{tabular}

and $N(i, j)=0$ if one of $i, j$ is 0 or 1 and the other is great than 4 .

In the case $i \geq 2$, we count the number of $K$-paths from $A_{i}$ to $B_{j}$ according to its intersection with the line $y=-2 x$. From Figure 3 , we see that there are 4 possible intersection points. We have

$$
\begin{aligned}
N(i, j)=B(2 j-i+2, & 2 i-j-4)+3 B(2 j-i+1,2 i-j-2) \\
+ & 3 B(2 j-i, 2 i-j)+B(2 j-i-1,2 i-j+2),
\end{aligned}
$$

where $B(a, b)=\left(\begin{array}{c}a+b \\ b\end{array}\right)$.

Let $M(a, b)$ be defined by

$$
M(a, b)=B(a+2, b-4)+3 B(a+1, b-2)+3 B(a, b)+B(a-1, b+2) .
$$

Then $N(i, j)=M(2 j-i, 2 i-j)$. We need to show that $M(a, b)=M(b, a)$ for $a+b \geq 2$, which implies $N(i, j)=N(j, i)$ for $i \geq 2$. Using the basic identity of binomial coefficients $B(c, d)=B(c-1, d)+B(c, d-1)$ for all integers $c$ and $d$, when $a+b \geq 2$, we have

$$
\begin{aligned}
M(a, b)=B(a-4, b+2)+ & 3 B(a-3, b+1)+6 B(a-2, b)+7 B(a-1, b-1) \\
+ & 6 B(a, b-2)+3 B(a+1, b-3)+B(a+2, b-4) .
\end{aligned}
$$


In the following Figure 4, every number we put at a point is the sum of the numbers at points that are to the left of it or under it. This corresponds to the formula $B(c, d)=$ $B(c-1, d)+B(c, d-1)$.

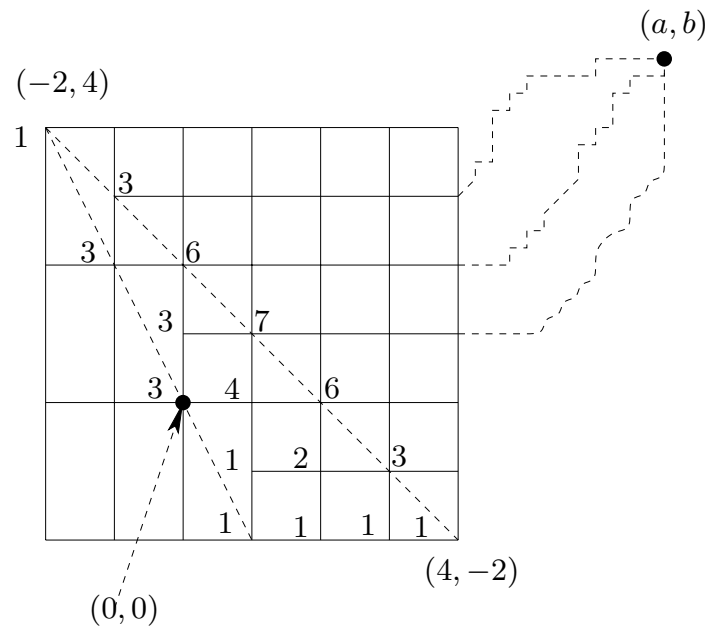

Figure 4: Proof of equation (66) by picture.

By the symmetry property $B(c, d)=B(d, c)$, we have $M(a, b)=M(b, a)$. This completes the proof.

Remark 6.5. Observe that the symmetry property of the numbers $(1,3,6,7,6,3,1)$ along the diagonal in Figure 4 implies (66). A bijective proof of this symmetry will induce a bijective proof of $N(i, j)=N(j, i)$, and then a bijective proof of Lemma 6.4.

Proof of Theorem 6.3. Let $G(m, n)$ be the number of $K$-paths starting at $(-2 m,-2 m)$ and ending at $(2 n, 2 n)$. We will prove that $G(m-1, n+1)=G(m, n)$ for all $m>0$. Then by induction, $G(m, n)=G(0, m+n)=a_{m+n}$.

We give the bijection as follows. Given a $K$-path $P$ from $(-2 m,-2 m)$ to $(2 n, 2 n)$, we separate it by the two lines $y+2=-2(x+2)$ and $x=-2 y$ into three parts: a $V_{2}$-path $P_{1}$, followed by a $K$-path $P_{2}$, followed by an $H_{2}$-path $P_{3}$.

Applying the bijection in the sliding lemma (Lemma 6.4) for $P_{2}$, we get $P_{2}^{\prime}$, a $K$-path starting on the line $y=-2 x$, ending on the line $x-2=-2(y-2)$. Then $P^{\prime}=P_{1} P_{2}^{\prime} P_{3}$ with starting point $(-2 m+2,-2 m+2)$ is the desired $K$-path.

A similar argument gives the inverse bijection.

This bijective proof of Theorem 6.3 is not ideal, though it is sufficient to prove the determinant formula (63). The proof relies on the sliding lemma, whose proof involves a case by case bijection that is not explicitly given. We would prefer a natural bijection for the sliding lemma that preserves the nonintersecting properties of $K$-paths. This is because such a bijection would give rise to a bijection from $\mathcal{U}_{K}(n)$ to $\mathcal{U}_{L}(n)$ : we could slide the $n$-tuples of $K$-paths in $\mathcal{U}_{K}(n)$ so that all the paths are above the line $x=-2 y$. Then the resulting paths would all be $H_{2}$-paths that can be easily converted into normal paths in $\mathcal{U}_{L}(n)$. 


\section{Trinomial Coefficients and $a_{n}$}

In this section, we introduce $T$-paths that are counted by trinomial coefficients. The trinomial coefficient $T(a, b)$ is defined by

$$
T(a, b)=\left[x^{a} y^{b}\right]\left(x^{2}+x y+y^{2}\right)^{\frac{a+b}{2}},
$$

if $a+b$ is even, and $T(a, b)=0$ otherwise.

The trinomial coefficients $T(a, b)$ have a simple combinatorial interpretation: We call a path $P$ a $T$-path if each step of $P$ is $(2,0)$ or $(1,1)$ or $(0,2)$. Then the number of $T$-paths that start at $(0,0)$ and end at $(a, b)$ is $T(a, b)$. This follows easily from the definition of $T(a, b)$. See the following Figure 5 , in which dots represent vertices of $T$-paths.

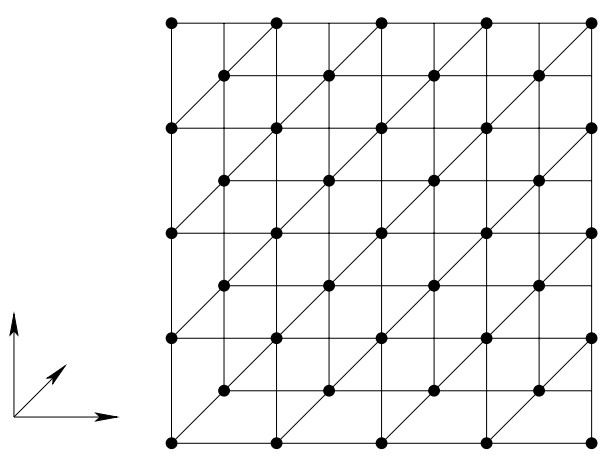

Figure 5: The grid for T-paths.

Using $T$-paths, we can give a better bijective proof of the sliding lemma. In addition, we find a new determinant identity (68).

Let $n=(a+b) / 2$. We can get another formula for $T(a, b)$ in the following way. We write $\left(x^{2}+x y+y^{2}\right)^{n}=\left(x(x+y)+y^{2}\right)^{n}$ and use the binomial theorem twice:

$$
\begin{aligned}
\left(x(x+y)+y^{2}\right)^{n} & =\sum_{k=0}^{n}\left(\begin{array}{l}
n \\
k
\end{array}\right) y^{2(n-k)} x^{k} \sum_{l=0}^{k}\left(\begin{array}{l}
k \\
l
\end{array}\right) x^{l} y^{k-l} \\
& =\sum_{a=0}^{2 n}\left(\sum_{k=0}^{a}\left(\begin{array}{l}
n \\
k
\end{array}\right)\left(\begin{array}{c}
k \\
a-k
\end{array}\right)\right) x^{a} y^{2 n-a} .
\end{aligned}
$$

So

$$
T(a, b)=\sum_{k=0}^{a}\left(\begin{array}{l}
n \\
k
\end{array}\right)\left(\begin{array}{c}
k \\
a-k
\end{array}\right) .
$$

This algebraic fact gives another combinatorial explanation of $T(a, b)$ :

Lemma 7.1. The number of paths from $(0,-2 m)$ to $(i,-i)$, in which the part below the line $y=-2 x$ is a $V_{2}$ path, and the other part is a normal path, is equal to the number of $T$-paths from $(0,-2 m)$ to $(i,-i)$, which is $T(i, 2 m-i)$. 
Proof. For a given path $P$ from $(0,-2 m)$ to $(i,-i)$, with the part $P_{1}$ below the line $y=-2 x$ a $V_{2}$ path, and the other part $P_{2}$ a normal path, it is clear that $P_{1}$ must end at a point $(j,-2 j)$ for some $j \geq 0$, and this $j$ is unique.

We observe that the number of horizontal steps in $P_{1}$ is $j$, which equals the total number of steps in $P_{2}$. Therefore, we can associate to each horizontal step in $P_{1}$ a step in $P_{2}$, with order preserved. We call this new path $Q$. Clearly, $Q$ is a $T$-path, since each step of $Q$ is a $(0,2)$-step, which is kept from $P_{1}$, or a $(1,1)$-step, by associating a vertical step in $P_{2}$ to a horizontal step in $P_{1}$, or a $(2,0)$-step, by associating a horizontal step in $P_{2}$ to a horizontal step in $P_{1}$. Since the above procedure is a rearrangement of the steps in $P_{1} P_{2}, Q$ is a $T$-path from $(0,-2 m)$ to $(i,-i)$. So $Q$ is the desired $T$-path. The above procedure is clearly reversible.

By reflecting in $y=-x$, we get

Lemma 7.2. The number of paths from $(i,-i)$ to $(0,2 m)$, in which the part above the line $x=-2 y$ is an $\mathrm{H}_{2}$ path, and the other part is a normal path, is also $T(i, 2 m-i)$.

Pictures for generalizations of these lemmas can be found in Figures 8 and 9. These lemmas correspond to the case $r=2$.

Theorem 7.3. The number of $K$-paths from $(0,-2 m)$ to $(2 n, 0)$ is equal to the number of $T$-paths from $(0,-2 m)$ to $(2 n, 0)$, which is $T(2 n, 2 m)$. Moreover, we have the following identity.

$$
U_{n}=\operatorname{det}(T(2 i, 2 j))_{0 \leq i, j \leq n-1} .
$$

Proof of Theorem 7.3. We can split any $K$-path from $(0,-2 m)$ to $(2 n, 0)$ into two parts: one ends at $(i,-i)$ and the other starts at $(i,-i)$ for some $i \geq 0$ (this $i$ is unique). Then using the two bijections in Lemmas 7.1 and 7.2, we have a bijective proof of the first part of the corollary.

We have shown in last section that $U_{n}$ equals the number of $n$-tuples of nonintersecting $K$-paths from $P_{0}, \ldots, P_{n-1}$ to $Q_{0}, \ldots, Q_{n-1}$, where $P_{i}=(-2 i,-2 i)$, and $Q_{i}=(2 i, 2 i)$ for $i=0,1, \ldots, n-1$. It is clear (see Figure 2 ) that it is still true if we replace $P_{i}$ by $\tilde{P}_{i}=(0,-2 i)$, and $Q_{i}$ by $\tilde{Q}_{i}=(2 i, 0)$. But from the first part of this corollary, the number of $K$-paths from $\tilde{P}_{i}$ to $\tilde{Q}_{j}$ is $T(2 j, 2 i)=T(2 i, 2 j)$ for all $0 \leq i, j \leq$. Then the identity (68) follows.

Remark 7.4. The identity (68) has a generalization in Section 8. Note that $U_{n}$ does not equal the number of $n$-tuples of nonintersecting $T$-paths from $P_{0}^{\prime}, \ldots, P_{n-1}^{\prime}$ to $Q_{0}^{\prime}, \ldots, Q_{n-1}^{\prime}$, because their steps can cross without meeting at a vertex of the T-paths.

Definition 7.5. We call a path $P$ a KT-path if it satisfies the following conditions.

1. The path $P$ never goes above the diagonal.

2. The part of $P$ that is to the left of the line $x=0$ is a $V_{2}$ path.

THE ELECTRONIC JOURNAL OF COMBINATORICS 13 (2006), \#R53 
3. The part of $P$ in the fourth quadrant is a T-path.

4. The part of $P$ that is above the line $y=0$ is an $\mathrm{H}_{2}$ path.

Theorem 7.6. The number of KT-paths from $(-2 m,-2 m)$ to $(2 n, 2 n)$ is $a_{m+n}$ for all $m+n \geq 0$.

We give three bijective proofs of this theorem. The first bijective proof establishes the bijection from $K$-paths to $K T$-paths. A sliding lemma for $K T$-paths will then yield a sliding lemma for $K$-paths. We find that it is much easier to slide $K T$-paths: we can slide slowly and we can also slide fast. We give the fast sliding in our bijection from $K T$-paths to $V_{2}$-paths. This is the second proof. The slow sliding will be given in Section 8 in a more general setting. This yields the third proof. We suspect that the sliding lemma for $K$-paths resulting from our second and third bijections are natural, i.e., preserve the nonintersecting property.

Bijection from $K T$-paths to $K$-paths. We first uniquely separate $P$, according to its intersections with the lines $x=0$ and $y=0$, into three parts, a $V_{2}$-path $P_{1}$, followed by a $T$-path $P_{2}$, followed by an $H_{2}$-path $P_{3}$, such that $P_{1}$ ends with a horizontal step and $P_{3}$ starts with a vertical step, except that $P_{1}$ and $P_{3}$ may be empty.

From Theorem 7.3, we can get a $K$-path $P_{2}^{\prime}$ from $P_{2}$ without changing the starting and ending points. Then $P^{\prime}=P_{1} P_{2}^{\prime} P_{3}$ is the desired $K$-path. This procedure is clearly reversible.

The next proof relies highly on Lemmas 7.1 and 7.2. The bijection $\phi_{v}$ for Lemma 7.1 maps a $T$-path $P$ to a $V_{2}$-path $P_{V}$ followed by a normal path $P_{N}$, in which the number of horizontal steps in $P_{V}$ equals the total number of steps in $P_{N}$. Given the starting point $S(P)$ and ending point $E(P)$ of $P$, we can predict the position of $E\left(P_{V}\right)=S\left(P_{N}\right): E\left(P_{V}\right)$ must lie on the line with slope -2 and passing through the point $O$, which is determined by the conditions that $O \rightarrow S(P)$ is vertical and the slope of $O \rightarrow E(P)$ is -1 .

Similarly the bijection $\phi_{h}$ for Lemma 7.2 maps a $T$-path $P$ to a normal path $P_{N}$ followed by an $H_{2}$-path $P_{H}$ with similar properties.

Fast sliding bijection from $K T$-paths to $V_{2}$-paths. Let $P$ be a $K T$-path from $(-2 m,-2 m)$ to $(2 n, 2 n)$ with $m, n \geq 0$.

We first uniquely separate $P$, according to its intersections with the lines $x=0$ and $y=0$, into a $V_{2}$-path $P_{1}$, followed by a $T$-path $P_{2}$, followed by an $H_{2}$-path $P_{3}$, such that $P_{1}$ ends with a horizontal step and $P_{3}$ starts with a vertical step, except that $P_{1}$ and $P_{3}$ may be empty. We will map $P_{2} P_{3}$ to a $V_{2}$ path with the same starting and ending points.

Suppose $S\left(P_{2}\right)=(0,-i)$ and $E\left(P_{2}\right)=(j, 0)$. Obviously we can assume $j>0$ for otherwise $P_{2}$ is a $V_{2}$-path and $P_{3}$ is the empty path. Applying $\phi_{v}$ to $P_{2}$ gives us a $V_{2}$-path $P_{2 V}$ followed by a normal path $P_{2 N}$ with $S\left(P_{2 N}\right)=(a,-2 a+j)$ for some $a>0$. See Figure 6, where we did not draw the paths explicitly.

Draw a vertical line at $S\left(P_{2 N}\right)$, which intersects the diagonal at $(a, a)$. It is easy to check that the the total number of steps of $P_{2 N}$ is $a$. Factor the $H_{2}$-path $P_{3}$, according 


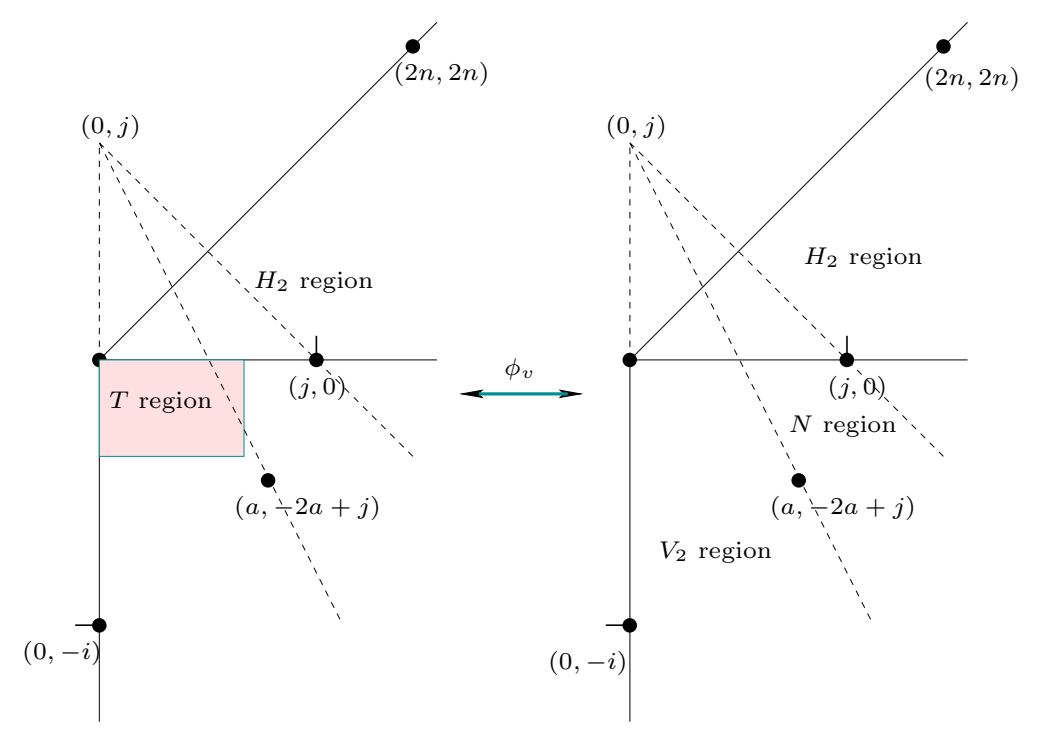

Figure 6: First step of the fast sliding.

to its intersection with the line $y=a$, into $P_{31} P_{3}^{\prime}$ such that $P_{3}^{\prime}$ starts with a vertical step. The the number of vertical steps of $P_{31}$ equals $a$. Applying $\phi_{h}^{-1}$ to $P_{2 N} P_{31}$ gives us a $T$-path $P_{2}^{\prime}$. See Figure 7 .

Now we are left to map $P_{2 V} P_{2}^{\prime} P_{3}^{\prime}$ to a $V_{2}$-path. If we slide down the path by $(a, a)$, then we met the same situation as for the path $P_{2} P_{3}$. Repeat the above procedure we can finally obtain the desired $V_{2}$-path. The procedure is reversible because of the required conditions of ending with a horizontal step or starting with a vertical step, as shown in Figures 6 and 7 .

\section{Generalizations of $K$-paths and $K T$-paths}

Let $g_{n}^{(r)}=\frac{1}{r n+1}\left(\begin{array}{c}(r+1) n \\ n\end{array}\right)$ be the number of $r+1$-ary trees with $n$ nodes, and

$$
g^{(r)}(x)=\sum_{n \geq 0} g_{n}^{(r)} x^{n}
$$

be the generating function. Then $g^{(r)}(x)$ satisfies the following functional equation.

$$
g^{(r)}(x)=1+x\left(g^{(r)}(x)\right)^{r+1} .
$$

For $r=1, g_{n}^{(1)}$ is the Catalan number. It is well-known that the Hankel determinants of the Catalan generating function are all 1 . We have studied the the case $r=2$. We wish to say something about the Hankel determinants of $g^{(r)}(x)$ for $r \geq 3$.

Since $H_{n}\left(g^{(r)}(x)\right)$ does not factor for $r \geq 3$, a formula like (4) is unlikely. However, we find generalizations of (63) (which is the same as (7)), (64), and (68). They are given 


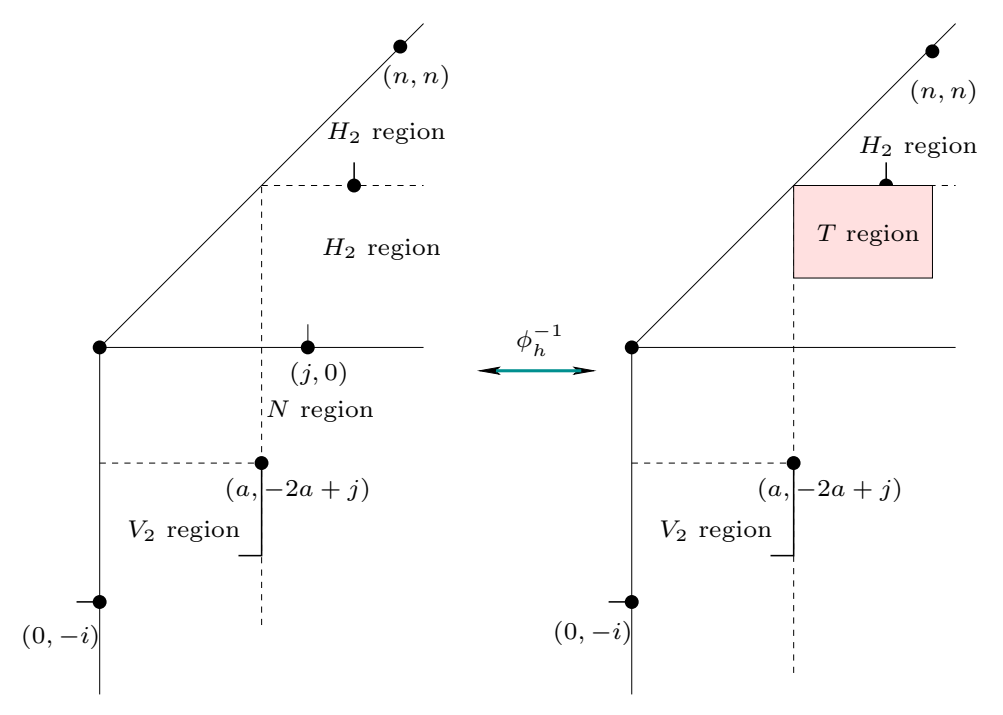

Figure 7: Second step of the fast sliding.

by (71), (72), and (73). Their algebraic proofs can be found in Section 9. Except for $H_{n}\left(g^{(r)}(x)\right)$, we do not have nonintersecting paths interpretation of these determinants.

We have natural generalizations of the concepts in the last two sections. A path is an $H_{r}$ path if each step is either $(r, 0)$ or $(0,1)$. Similarly, a path is a $V_{r}$ path if each step is either $(1,0)$ or $(0, r)$. The following is equivalent to a special case of a classical result given (without proof) by Barbier [4]. (See [12] for a new proof and further references.)

Proposition 8.1. The number of $V_{r}$-paths (or $H_{r}$-paths) from $(0,0)$ to $(r n, r n)$ that never go above the diagonal is $g_{n}^{(r)}$.

A path is a $T^{(r)}$-path if each of its step is $(r, 0),(r-1,1), \ldots$, or $(0, r)$. For any path $P$, we denote by $S(P)$ the starting point, $E(P)$ the ending point, and $L(P)$ the number of steps in $P$.

For a $T^{(r)}$-path $P$ with $S(P)=(0,0)$ and $L(P)=k, E(P)$ must lie on the line $y=-x+r k$. So to compute $L(P)$, we take the sum of the $x$-coordinate and $y$-coordinate of $E(P)-S(P)$, and divide by $r$. A normal path is also a $T^{(1)}$-path, a $V_{1}$ path, and an $H_{1}$ path, and a $T$-path is a $T^{(2)}$-path.

Let $T^{(r)}(a, b)$ be the number of $T^{(r)}$-paths from $(0,0)$ to $(a, b)$. Then $T^{(r)}(a, b)=0$ if $a+b$ is not divisible by $r$, so we can suppose $a=r i-s$ and $b=r j+s$ for some $i, j$ and $0 \leq s \leq r-1$. We have

$$
T^{(r)}(r i+s, r j-s)=\left[x^{r i+s} y^{r j-s}\right]\left(x^{r}+x^{r-1} y+\cdots+y^{r}\right)^{i+j} .
$$

Since the right-hand side of the above equation is homogeneous in $x$ and $y$, we can write it in terms of one variable $t$, where $t=y / x$.

Let $\alpha=1+t+t^{2}+\cdots+t^{r}$ and $\beta=\alpha / t^{r}=1+t^{-1}+\cdots t^{-r}$. Then

$$
T(r i+s, r j-s)=\mathrm{CT} t^{s} \alpha^{i} \beta^{j}=\mathrm{CT} t^{s-r j} \alpha^{i+j}
$$


where CT means to take the constant term of a Laurent polynomial of $t$.

Definition 8.2. We call a path $P$ a $K^{(r)}$-path if it satisfies the following conditions.

1. The path $P$ never goes above the diagonal.

2. The part of $P$ that is to the left of the line $y=-r x$ is a $V_{r}$ path.

3. The part of $P$ between the two lines $y=-r x$ and $x=-r y$ is a $T^{(r-1)}$-path.

4. The part of $P$ that is above the line $x=-r y$ is an $H_{r}$ path.

Definition 8.3. We call a path $P$ a $K T^{(r)}$-path if it satisfies the following conditions.

1. The path $P$ never goes above the diagonal.

2. The part of $P$ that is to the left of the line $x=0$ is a $V_{r}$ path.

3. The part of $P$ in the fourth quadrant is a $T^{(r)}$-path.

4. The part of $P$ that is above the line $y=0$ is an $H_{r}$ path.

For example, a $K$-path is a $K^{(2)}$-path, and a $K T$-path is a $K T^{(2)}$-path.

Let $\mathbb{K}(m, n, r)$ be the set of all $K^{(r)}$-paths from $(-m r,-m r)$ to $(n r, n r)$. Let $\mathbb{T}(m, n, r, s)$ be the set of all $K T^{(r)}$-paths from $(s-m r, s-m r)$ to $(n r+s, n r+s)$. Now we can state our main results.

Theorem 8.4. The cardinality of $\mathbb{K}(m, n, r)$ is $g_{n+m}^{(r)}$ for all $m$ and $n$. The cardinality of $\mathbb{T}(m, n, r, s)$ is also $g_{n+m}^{(r)}$ for all $m, n$ and $s$.

As in the case $r=2$, if $m \leq 0$ (or $n \leq 0$ ), then $K^{(r)}$-paths and $K T^{(r)}$-paths are in fact $H_{r}$-paths (or $V_{r}$-paths), and in these cases, Theorem 8.4 follows from Proposition 8.1. The idea of the proof of Theorem 8.4 is to show that $|\mathbb{T}(m, n, r, s)|=|\mathbb{T}(m, n, r, s-1)|$ for all $1 \leq s \leq r$. Then $|\mathbb{T}(m, n, r, s)|=g_{m+n}^{(r)}$ follows by induction. We will give a bijection from $\mathbb{T}(m, n, r, 0)$ to $\mathbb{K}(m, n, r)$.

The bijective proof we are going to give highly relies on the following lemma, especially on the bijection from $\mathbf{T}(i, j)$ to $\mathbf{V}(i, j)$, which is a generalization of Lemma 7.1.

Lemma 8.5. The following four sets all have cardinality $T^{(r)}(j, r i-j)$.

1. The set $\mathbf{T}(i, j)$ of all $T^{(r)}$-paths from $(0,-r i)$ to $(j,-j)$.

2. The set $\mathbf{T}^{\prime}(i, j)$ of all $T^{(r)}$-paths from $(j,-j)$ to $(r i, 0)$.

3. The set $\mathbf{V}(i, j)$ of all paths from $(0,-r i)$ to $(j,-j)$, with the part before the line $y=-r x$ a $V_{r}$ path, and the part after the line $y=-r x a T^{(r-1)}$-path.

4. The set $\mathbf{H}(i, j)$ of all paths from $(j,-j)$ to $($ ri, 0$)$ with the part before the line $x=$ -ry a $T^{(r-1)}$-path, and the part after the line $x=-r y$ an $H_{r}$ path. 
Proof. We construct only the bijection from $\mathbf{T}(i, j)$ to $\mathbf{V}(i, j)$. The bijection from $\mathbf{T}^{\prime}(i, j)$ to $\mathbf{H}(i, j)$ is similar. The bijection from $\mathbf{V}(i, j)$ to $\mathbf{H}(i, j)$ and the bijection from $\mathbf{T}(i, j)$ to $\mathbf{T}^{\prime}(i, j)$ are given by reflecting in the line $y=-x$.

For any given path $T \in \mathbf{T}(i, j)$ the steps in $T$ are $(r-k, k)$ for $k=0,1, \ldots, r$. We first replace all the steps in $T$ that are not $(0, r)$ with steps $(r, 0)$. Then we get a path $T_{1}$, with $E\left(T_{1}\right)$ on the line $y=-x$. Changing every $(r, 0)$-step in $T_{1}$ into $(1,0)$ will give us a $V_{r}$ path $V_{1}$ with $E\left(V_{1}\right)$ on the line $y=-r x$. By removing all the $(0, r)$-steps in $T$, and changing every $(r-k, k)$-step into $(r-k-1, k)$ for $k=0,1, \ldots r-1$, we get a $T^{(r-1)}$-path $V_{2}$. Then $V=V_{1} V_{2} \in \mathbf{V}(i, j)$ is the desired path. One way to see that $E(V)=(j,-j)$ is that if we regard an $(r-k, k)$-step as a $(1,0)$ step followed by a $(r-k-1, k)$-step for $k=0,1, \ldots, r-1$, then the bijection we gave is just a rearrangement of the steps in $T$.

The inverse procedure is as follows. For a given $V^{\prime} \in \mathbf{V}(i, j)$, the line $y=-r x$ divides $V^{\prime}$ into a $V_{r}$ path $V_{1}^{\prime}$ followed by a $T^{(r-1)}$ path $V_{2}^{\prime}$. Suppose $E\left(V_{1}^{\prime}\right)=\left(k^{\prime},-r k^{\prime}\right)$ for some $k^{\prime}$. We can see that the number of $(1,0)$-steps in $V_{1}^{\prime}$, which is $k^{\prime}$, equals the total number of steps in $V_{2}^{\prime}$, which is $\left(\left(j-k^{\prime}\right)+\left(-j+r k^{\prime}\right)\right) /(r-1)=k^{\prime}$. Then we can associate to each $(1,0)$ step in $V_{1}^{\prime}$ a step in $V_{2}^{\prime}$, with order preserved. This gives us a $T^{(r)}$-path $T^{\prime} \in \mathbf{T}(i, j)$. In Figure 8, we give an example of the case $r=4$. The above two procedures are clearly

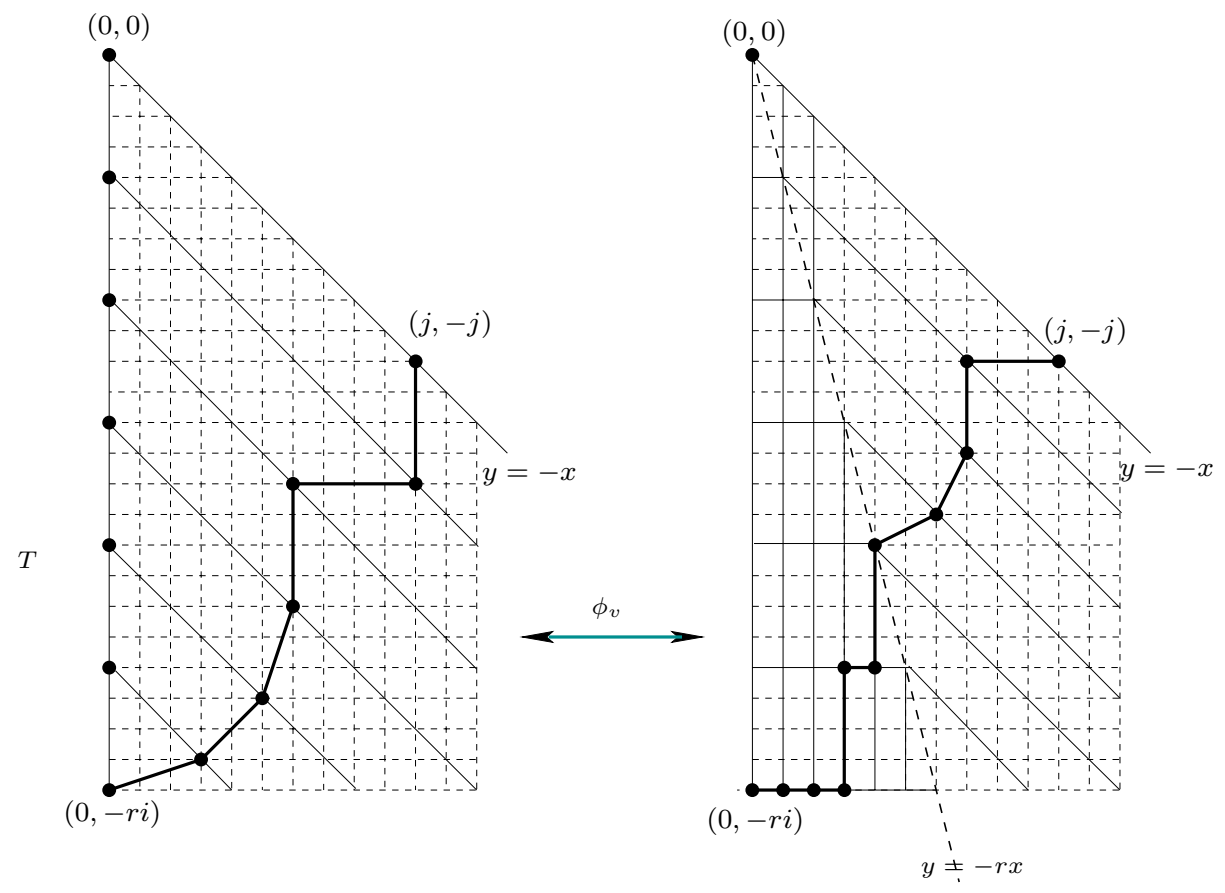

Figure 8: A $T^{(r)}$-path $T$ and its image under $\phi_{v}$.

inverse to each other.

Remark 8.6. The bijection from $\mathbf{T}(i, j)$ to $\mathbf{V}(i, j)$ we gave originated from the following algebraic fact.

For $r \geq 3$, there are many ways to group and expand the polynomial $\left(x^{r}+x^{r-1} y+\right.$ $\left.\cdots+y^{r}\right)^{m}$. We find the following way has a nice combinatorial explanation. 


$$
\begin{aligned}
\left(x\left(x^{r-1}+x^{r-2} y+\cdots y^{r-1}\right)+y^{r}\right)^{m} & =\sum_{j=0}^{m}\left(\begin{array}{c}
m \\
j
\end{array}\right) y^{r(m-j)} x^{j}\left(x^{r-1}+x^{r-2} y+\cdots y^{r-1}\right)^{j} \\
& =\sum_{i=0}^{r m}\left(\sum_{j=0}^{m}\left(\begin{array}{c}
m \\
j
\end{array}\right) T^{(r-1)}(r j-i, i-j)\right) x^{i} y^{r m-i}
\end{aligned}
$$

So we have

$$
T^{(r)}(i, r m-i)=\sum_{j=0}^{m}\left(\begin{array}{c}
m \\
j
\end{array}\right) T^{(r-1)}(r j-i, i-j) .
$$

In Figure 8, $|\mathbf{V}(i, j)|$ can be counted according to the intersection points of the paths in $\mathbf{V}(i, j)$ with the line $y=-r x$. This yields (70).

We denote the bijection from $\mathbf{T}(i, j)$ to $\mathbf{V}(i, j)$ by $\phi_{v}$, and the bijection from $\mathbf{T}^{\prime}(i, j)$ to $\mathbf{H}(i, j)$ by $\phi_{h}$. One thing we should mention is that neither $\phi_{h}$ nor $\phi_{v}$ changes the starting point and the ending point. The path in Figure 9 is obtained from the $T^{(r)}$-path $T$ in Figure 8 by applying $\phi_{h}$.

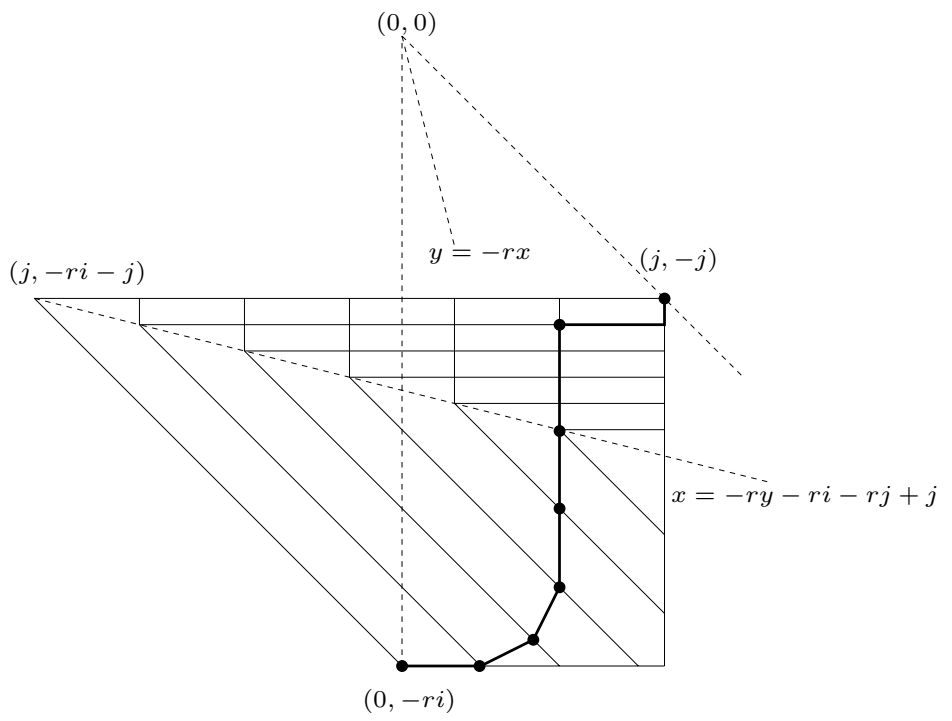

Figure 9: The image of $T$ under $\phi_{h}$.

Applying $\phi_{v}$ to a $T^{(r)}$ path will give us a $V_{r}$ path followed by a $T^{(r-1)}$ path, in which the number of horizontal steps in the $V_{r}$ path equals the total number of steps in the $T^{(r-1)}$ path. We can locate the ending point of the $V_{r}$ path by the following three easy steps. (See Figure 8.)

1. Draw a vertical line at $S(T)$. 
2. Draw a line of slope -1 at $E(T)$.

3. At the intersecting point of the above two lines, draw a line of slope $-r$. Then this is the line on which the ending point of the $V_{r}$ path must lie.

We call the line obtained in the above three steps the bisecting line $\mathrm{BL}_{v}(T)$ of $\phi_{v}(T)$. For any $T^{(r)}$ path $T$, with $S(T)=(0,-r i)$ and $E(T)=(j,-j), \mathrm{BL}_{v}(T)$ is $y=-r x$, which is independent of $i$ and $j$.

A similar argument for $\phi_{h}$ holds for a $T^{(r)}$-path $T^{\prime}$. The corresponding three steps are given as follows. (See Figure 9.)

1. Draw a horizontal line at $E\left(T^{\prime}\right)$.

2. Draw a line of slope -1 at $S\left(T^{\prime}\right)$.

3. At the intersecting point of the above two lines, draw a line of slope $-1 / r$. Then this is the line on which the starting point of the $H_{r}$-path must lie.

We call the line obtained in the above three steps the bisecting line $\mathrm{BL}_{h}\left(T^{\prime}\right)$ of $\phi_{h}\left(T^{\prime}\right)$.

The following is a generalization of Theorem 7.3.

Lemma 8.7. The number of each of the following four kinds of paths from $(0,-r i+s)$ to $(r j+s, 0)$ equals to $T(r j+s, r i-s)$.

1. All $T^{(r)}$-paths from $(0,-r i+s)$ to $(r j+s, 0)$.

2. All paths from $(0,-r i+s)$ to $(r j+s, 0)$ consisting of a $V_{r}$ path, followed by a $T^{(r-1)}$ path, in which the number of $(1,0)$-steps in the $V_{r}$ path equals the total number of steps in the $T^{(r-1)}$ path.

3. All the paths from $(0,-r i+s)$ to $(r j+s, 0)$ consisting of a $T^{(r-1)}$ path, followed by an $H_{r}$ path, in which the number of $(0,1)$-steps in the $H_{r}$ path equals the total number of steps in the $T^{(r-1)}$ path.

4. All the paths from $(0,-r i+s)$ to $(r j+s, 0)$, with the part before the line $y=-r x+s$ a $V_{r}$ path, between the lines $y=-r x+s$ and $x=-r y+s a T^{(r-1)}$ path, and the part after the line $x=-r y+s$ an $H_{r}$ path.

Proof. Part 1 follows from the definition of $T^{(r)}(r j+s, r i-s)$. Part 2 and part 3 are obvious by Lemma 8.5, so we need only prove part 4.

For a given $T^{(r)}$ path $T$ from $(0,-r i+s)$ to $(r j+s, 0)$, we can uniquely separate it by the line $y=-x+s$ into a $T^{(r)}$ path $T_{1}$ followed by a $T^{(r)}$ path $T_{2}$. Applying $\phi_{v}$ on $T_{1}$, we get a $V_{r}$ path $T_{1 V}$ followed by a $T^{(r-1)}$ path $T_{1 T}$. Applying $\phi_{h}$ on $T_{2}$, we get a $T^{(r-1)}$ path $T_{2 T}$ followed by an $H_{r}$ path $T_{2 H}$. Using the three steps for finding $\mathrm{BL}_{v}\left(T_{1}\right)$, we see that $E\left(T_{1 V}\right)$ must lie on the line $y=-r x+s$, since the line $y=-x+s$ intersects the line $x=0$ at $(0, s)$. Similarly, $S\left(T_{2 T}\right)$ must lie on the line $x=-r y+s$, since the line $y=-x+s$ intersect the line $y=0$ at $(s, 0)$. Together with the fact that 
$E\left(T_{1 T}\right)=E\left(T_{1}\right)=S\left(T_{2}\right)=S\left(T_{2 T}\right)$, we see that $T_{1 T} T_{2 T}$ is also a $T^{(r-1)}$ path and the path $T_{1 V} T_{1 T} T_{2 T} T_{2 H}$ is the desired path.

The above procedure is clearly invertible.

The bijection in the above proof will induce a bijection from $\mathbb{T}(m, n, r, 0)$ to $\mathbb{K}(m, n, r)$. We will see this in the proof of Theorem 8.4.

For any $1 \leq s \leq r$, let $\mathbb{A}_{s}(i, j)$ be the set of $K T^{(r)}$-paths from $(0,-(r i-s))$ to $(r j+s, 1)$, and $\mathbb{B}_{s}(i, j)$ be the set of $K T^{(r)}$-paths from $(-1,-(r i-s+1)$ to $(r j+s-1,0)$. Then we have the following lemma, which will induce the bijection from $\mathbb{T}(m, n, r, s)$ to $\mathbb{T}(m, n, r, s-1)$.

Lemma 8.8 (Slow Sliding Lemma). There is a bijection from $\mathbb{A}_{s}(i, j)$ to $\mathbb{B}_{s}(i, j)$ for all $i, j$.

We will give two proofs for this lemma. The algebraic proof will be given in the next section. The bijective proof is as follows.

Bijective proof of Lemma 8.8. For any given $P \in \mathbb{A}_{s}(i, j)$, we uniquely factor $P$, according to its intersections with the lines $y=0$ and $y=-x+s$, into $P_{1} P_{2} P_{3}$, where we require $P_{3}$ to start with a vertical step. In the left picture of Figure 10, we marked each intersection point by a $\circ$.

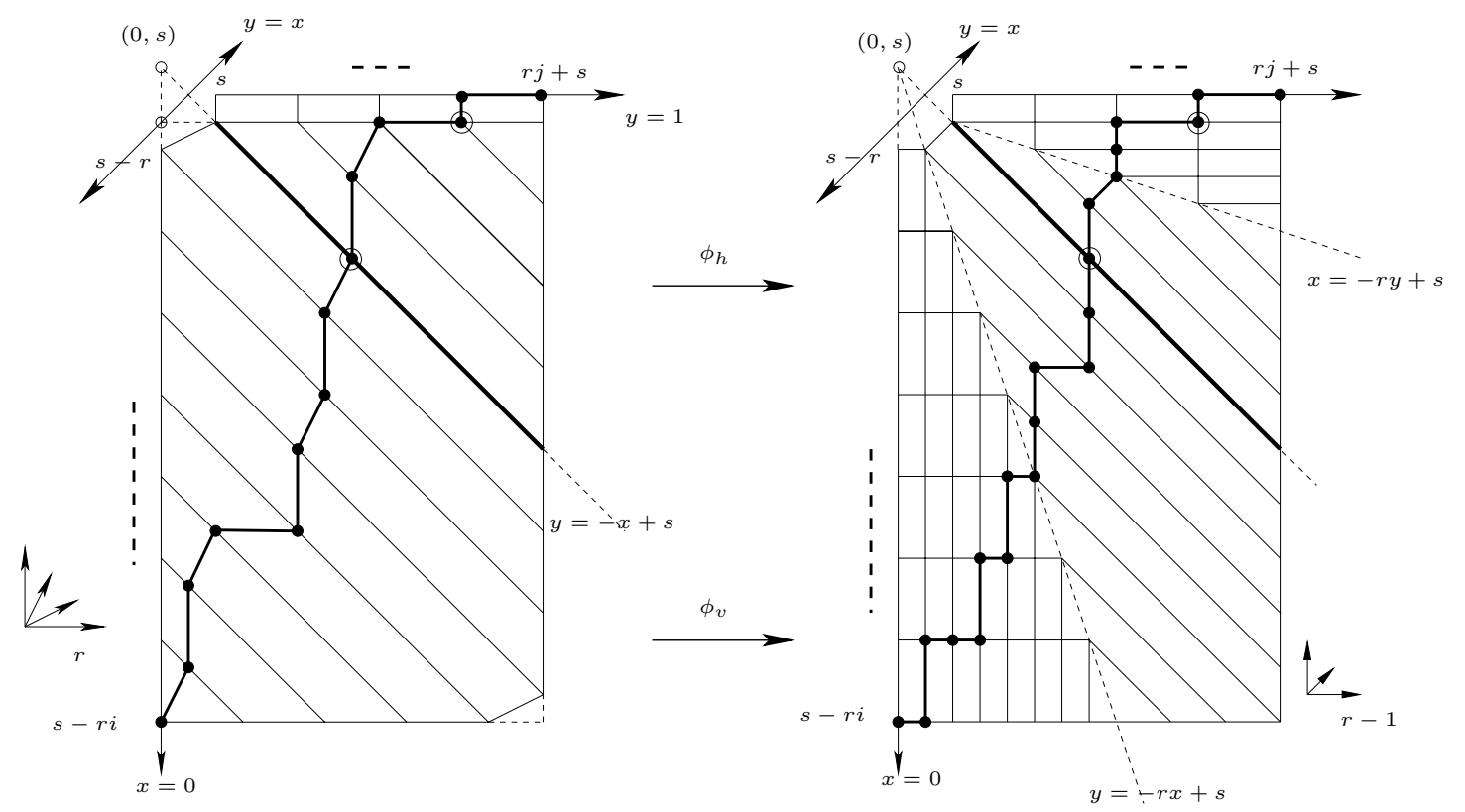

Figure 10: First step of the slow sliding bijection.

Now we apply $\phi_{v}$ to $P_{1}$ to obtain a $V_{r}$-path followed by a $T^{(r-1)}$-path, and apply $\phi_{h}$ to $P_{2}$ to obtain a $T^{(r-1)}$-path followed by an $H_{r}$-path. The bisection lines are $y=-r x+s$ and $x=-r y+s$, as drawn in the right picture of Figure 10. 
Denote by $P^{\prime}$ the whole path obtained this way. We uniquely factor $P^{\prime}$, according to its intersections with the lines $x=1$ and $y=-x+s-r+1$ into $P_{1}^{\prime} P_{2}^{\prime} P_{3}^{\prime}$, where we require $P_{1}^{\prime}$ to end with a horizontal step. In the left picture of Figure 11, we marked each intersection point by a $\square$.

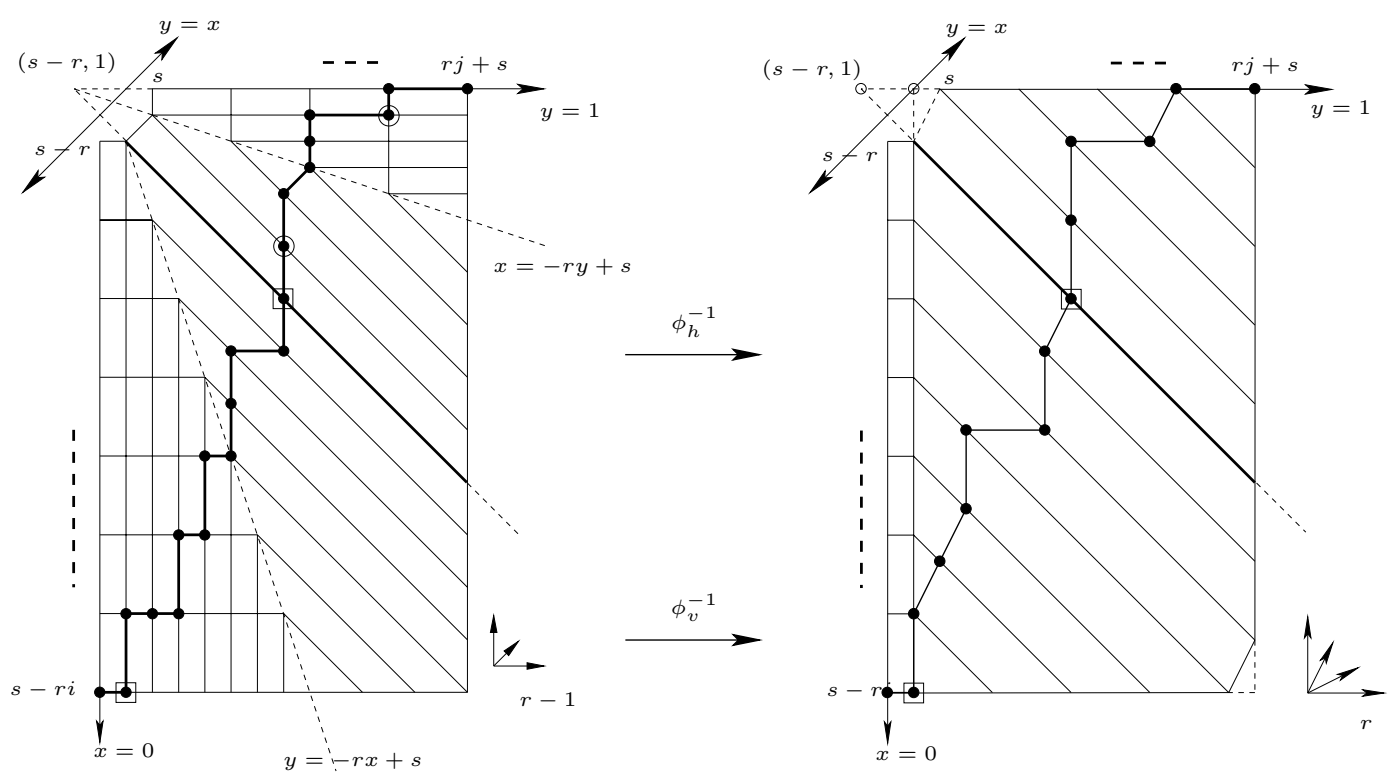

Figure 11: Second step of the slow sliding bijection.

Now we apply $\phi_{v}^{-1}$ to $P_{2}^{\prime}$ to obtain a $T^{(r)}$-path $Q_{2}$, and apply $\phi_{h}^{-1}$ to $P_{3}^{\prime}$ to obtain a $T^{(r)}$-path $Q_{3}$. See the right picture of Figure 11. We need to check that the three lines $y=-x+s-r+1, x=-r y+s$, and $y=1$ intersect at the point $(s-r, 1)$, so that $\phi_{h}^{-1}\left(P_{3}^{\prime}\right)$ is well defined.

Finally, let $Q$ be obtained from $P_{1}^{\prime} Q_{2} Q_{3}$ by sliding down by $(1,1)$. Then $Q$ is the desired path. Every step in the above procedure is invertible. This completes the proof.

Proof of Theorem 8.4. First we construct the bijection from $\mathbb{T}(m, n, r, 0)$ to $\mathbb{K}(m, n, r)$. This shows that $|\mathbb{T}(m, n, r, 0)|=|\mathbb{K}(m, n, r)|$.

Recall that any $P \in \mathbb{T}(m, n, r, 0)$ is a $K T^{(r)}$-path from $(-r m,-r m)$ to $(r n, r n)$, and any $Q \in \mathbb{K}(m, n, r)$ is a $K_{r}$-path with the same ending points. We can uniquely factor $P$, according to its intersections with the lines $x=0$ and $y=0$, into $P_{1} P_{2} P_{3}$, such that $P_{1}$ is a $V_{r}$ path ending with a horizonal step and $P_{3}$ is an $H_{r}$ path starting with a vertical step, except that $P_{1}$ and $P_{3}$ may be empty. Applying the bijection of Lemma 8.7 part 4 to $P_{2}$, we get a $K_{r}$-path $Q_{2}=Q_{2 a} Q_{2 b} Q_{2 c}$ from $S\left(P_{2}\right)$ to $E\left(P_{2}\right)$, with $Q_{2 a}$ a $V_{r}$ path ending on the line $y=-r x, Q_{2 b}$ a $T^{(r-1)}$ path ending on the line $x=-r y$, and $Q_{2 c}$ an $H_{r}$ path. Then $P_{1} Q_{2 a}$ is a $V_{r}$ path, and $Q_{2 c} P_{3}$ is an $H_{r}$ path. So $Q=P_{1} Q_{2} P_{3}=\left(P_{1} Q_{2 a}\right) Q_{2 b}\left(Q_{2 c} P_{3}\right)$ belonging to $\mathbb{K}(m, n, r)$ is the desired path. The above procedure is clearly reversible.

Next we construct the bijection from $\mathbb{T}(m, n, r, s)$ to $\mathbb{T}(m, n, r, s-1)$ for $1 \leq s \leq r$, which implies $|\mathbb{T}(m, n, r, s)|=|\mathbb{T}(m, n, r, s-1)|$. Any $P \in \mathbb{T}(m, n, r, s)$ can be uniquely 
factored, according to its intersections with the lines $x=0$ and $y=1$, into $P_{1} P_{2} P_{3}$, such that $P_{1}$ is a $V_{r}$ path ending with a horizonal step and $P_{3}$ is an $H_{r}$ path starting with a vertical step, except that $P_{1}$ and $P_{3}$ may be empty. Then $P_{2}$ is a $K T^{(r)}$-path.

Applying the bijection of Lemma 8.8 to $P_{2}$, we get a $K T^{(r)}$-path $Q_{2}=Q_{2 a} Q_{2 b}$, with $Q_{2 a}$ a $V_{r}$ path starting on the line $x=-1$ and ending on the line $x=0$, and $Q_{2 b}$ a $T_{r}$ path ending on the line $y=0$. Let $Q_{1}$ be obtained from $P_{1}$ by sliding down by $(1,1)$, and $Q_{3}$ be obtained from $P_{3}$ by sliding down by $(1,1)$. Then $Q=Q_{1} Q_{2} Q_{3}=\left(Q_{1} Q_{2 a}\right) Q_{2 b} Q_{3}$ is a path from $(s-1-m r, s-1-m r)$ to $(s-1+n r, s-1, n r)$ that never goes above the diagonal and with the part before the line $x=0$ a $V_{r}$ path, the part between the two lines $x=0$ and $y=0$ a $T_{r}$ path, and the part after the line $y=0$ an $H_{r}$ path. Hence $Q \in \mathbb{T}(m, n, r, s-1)$ is the desired path. The above procedure is clearly reversible.

Finally, we use induction to conclude the theorem. By the second part, it is easy to see that $|\mathbb{T}(m, n, r, s)|=|\mathbb{T}(m, n, r, r)|$ for $0 \leq s \leq r$. But $\mathbb{T}(m, n, r, r)$ is in fact $\mathbb{T}(m-1, n+1, r, 0)$. Theorem 8.4 then follows by induction and the fact that $\mid \mathbb{T}(0, m+$ $n, r, 0) \mid=g_{m+n}^{(r)}$.

Remark 8.9. We can also give a fast sliding bijection from $\mathbb{T}(m, n, r, 0)$ to $\mathbb{T}(0, m+$ $n, r, 0)$.

Recall that $g^{(r)}(x)$ is the generating function of $r+1$-ary trees. Let $f^{(r)}(x)=g^{(r)}(x)-1$. Then $f^{(r)}$ satisfies the following functional equation.

$$
f^{(r)}=x\left(1+f^{(r)}\right)^{r+1} .
$$

If we count $K^{(r)}$-paths from $(-m r,-m r)$ to $(n r, n r)$ according to their intersections with the lines $y=-r x$ and $x=-r y$, we see that Theorem 8.4 yields the matrix identity

$$
\begin{aligned}
\left(g_{i+j}^{(r)}\right)_{0 \leq i, j \leq n-1}=\left(\left[x^{i}\right] g^{(r)}\left(f^{(r)}\right)^{j}\right)_{0 \leq i, j \leq n-1} & \\
& \left(T^{(r-1)}(r j-i, r i-j)\right)_{0 \leq i, j \leq n-1}\left(\left[x^{j}\right] g^{(r)}\left(f^{(r)}\right)^{i}\right)_{0 \leq i, j \leq n-1},
\end{aligned}
$$

where

$$
\left[x^{i}\right] g^{(r)}\left(f^{(r)}\right)^{j}=\frac{(r+1) j+1}{(r+1) i+1}\left(\begin{array}{c}
(r+1) i+1 \\
i-j
\end{array}\right)
$$

is the number of $V_{r}$-paths from $(-r i,-r i)$ to $(j,-r j)$ that never go above the diagonal.

Since the transformation matrices in (71) are upper (or lower) triangular matrices with diagonal entries 1, we have

$$
\operatorname{det}\left(g_{i+j}^{(r)}\right)_{0 \leq i, j \leq n-1}=\operatorname{det}\left(\left(T^{(r-1)}(r j-i, r i-j)\right)_{0 \leq i, j \leq n-1}\right.
$$

A similar argument gives

$$
\operatorname{det}\left(g_{i+j}^{(r)}\right)_{0 \leq i, j \leq n-1}=\operatorname{det}\left(T^{(r)}(r i, r j)\right)_{0 \leq i, j \leq n-1}=\operatorname{det}\left(T^{(r)}(r i-s, r j+s)\right)_{0 \leq i, j \leq n-1},
$$

for any $0 \leq s \leq r-1$ 


\section{The Algebraic Proof}

The scheme of our algebraic proof of Lemma 8.8 is by first representing our object as the constant term of a rational function, and then evaluating the constant term. This technique is well-known. For instance, Egorychev [7] gave many applications for evaluating combinatorial sums in the context of residues (equivalent to constant terms). We also use this method, together the method in Section 4, to give algebraic proofs of (72) and (73).

The only thing we need here is the following Proposition 9.1. Its proof is included since the idea of the proof applies to most of our examples. We will give a different algebraic proof of equations (48) and (52).

Let $B(x, y, t) \in \mathbb{Q}\left[t, t^{-1}\right][[x, y]]$. Then $B(x, y, t)$ can be written as

$$
B(x, y, t)=\sum_{i, j=0}^{\infty} b_{i j}(t) x^{i} y^{j}
$$

where $b_{i j}(t) \in \mathbb{Q}\left[t, t^{-1}\right]$. Define

$$
\mathrm{CT} B(x, y, t)=\sum_{i, j=0}^{\infty}\left(\mathrm{CT} b_{i j}(t)\right) x^{i} y^{j}
$$

where $\mathrm{CT} b_{i j}(t)$ is the constant term of the Laurent polynomial $b_{i j}(t)$ in $t$.

The general problem in this section is to find the constant term of the function $(1-$ $\left.P\left(t^{-1}\right) x\right)^{-1}(1-Q(t) y)^{-1}$, for some specific $P(t), Q(t) \in \mathbb{Q}[t]$.

Proposition 9.1. Let $P(t)$ and $Q(t)$ be polynomials in $t$, and let $a_{m n}=\operatorname{CT~} P(t)^{m} Q\left(t^{-1}\right)^{n}$. Then

$$
\sum_{m, n} a_{m n} x^{m} y^{n}
$$

is a rational function in $x$ and $y$.

Proof. We show that

$$
\mathrm{CT} \frac{1}{(1-P(t) x)\left(1-Q\left(t^{-1}\right) y\right)}
$$

is rational, where we work in the ring $\mathbb{Q}\left[t, t^{-1}\right][[x, y]]$. We may assume that $P(t)$ has degree at at least 1 . Let $d$ be the degree of $Q(t)$. Let

$$
F=\frac{1}{(1-P(t) x)\left(1-Q\left(t^{-1}\right) y\right)}=\frac{t^{d}}{(1-P(t) x)\left(t^{d}-t^{d} Q\left(t^{-1}\right) y\right)} .
$$

Since $t^{d} Q\left(t^{-1}\right)$ is a polynomial in $t$ of degree at most $d$, and the degree of $P(t)$ is at least $1, F$ has a partial fraction expansion in $t$ that may be written

$$
F=\frac{1}{R(x, y)}\left(\frac{A(x, y, t)}{1-P(t) x}+\frac{B(x, y, t)}{t^{d}-t^{d} Q\left(t^{-1}\right) y}\right)=\frac{1}{R(x, y)}\left(\frac{A(x, y, t)}{1-P(t) x}+\frac{B(x, y, t) t^{-d}}{1-Q\left(t^{-1}\right) y}\right)
$$

THE ELECTRONiC JOURNAL OF COMBINATORICS 13 (2006), \#R53 
where $R(x, y)$ is a polynomial in $x$ and $y, A(x, y, t)$ and $B(x, y, t)$ are polynomials in $x$, $y$, and $t$, and the degree of $B$ in $t$ is less than $d$.

Now the constant term in $t$ of $B(x, y, t) t^{-d} /\left(1-Q\left(t^{-1}\right) y\right)$ is 0 and the constant term in $A(x, y, t) /(1-P(t) x)$ is $A(x, y, 0) /(1-P(0) x)$. We would like to conclude that

$$
\mathrm{CT} F=\frac{A(x, y, 0)}{R(x, y)(1-P(0) x)} .
$$

However, we don't know that $1 / R(x, y)$ has a power series expansion. To avoid this problem, we multiply $(74)$ by $R(x, y)$ to get

$$
R(x, y) F=\frac{A(x, y, t)}{1-P(t) x}+\frac{B(x, y, t) t^{-d}}{1-Q\left(t^{-1}\right) y} .
$$

Then

$$
\mathrm{CT} R(x, y) F=\frac{A(x, y, 0)}{1-P(0) x}
$$

but since $\mathrm{CT} R(x, y) F=R(x, y) \mathrm{CT} F$, (75) follows.

Since the main idea of this proof is a partial fraction decomposition, we call this method the partial fraction method. In the following examples, we use formula (69). Let $\alpha=1+t+t^{2}+\cdots+t^{r}$ and $\beta=\alpha / t^{r}=1+t^{-1}+\cdots t^{-r}$. Then

$$
T(r i+s, r j-s)=\mathrm{CT} t^{s} \alpha^{i} \beta^{j}
$$

In particular,

$$
\left(\begin{array}{c}
m+n \\
m
\end{array}\right)=\left[t^{n}\right](1+t)^{m+n}=\mathrm{CT}(1+t)^{m}\left(1+\frac{1}{t}\right)^{n} .
$$

Example 9.2. A different proof of identity (48)

$$
\frac{1-x y}{1-x y^{2}-3 x y-x^{2} y}=\sum_{i, j}\left(\begin{array}{c}
i+j \\
2 i-j
\end{array}\right) x^{i} y^{j} .
$$

Proof. We have

$$
\begin{aligned}
\sum_{i, j \geq 0}\left(\begin{array}{c}
i+j \\
2 i-j
\end{array}\right) x^{i} y^{j} & =\sum_{i, j \geq 0}\left(\mathrm{CT}(1+t)^{2 i-j}\left(1+t^{-1}\right)^{2 j-i}\right) x^{i} y^{j} \\
& =\mathrm{CT} \sum_{i, j \geq 0} \frac{(1+t)^{2 i}}{\left(1+t^{-1}\right)^{i}} x^{i} \cdot \frac{\left(1+t^{-1}\right)^{2 j}}{(1+t)^{j}} y^{j} \\
& =\mathrm{CT} \frac{1}{\left(1-t x-t^{2} x\right)\left(1-t^{-1} y-t^{-2} y\right)}
\end{aligned}
$$


Using Maple, we find the partial fraction expansion in $t$ :

$$
\begin{aligned}
\frac{1}{\left(1-t x-t^{2} x\right)\left(1-t^{-1} y-t^{-2} y\right)}= & \frac{1}{1-3 x y-x^{2} y-x y^{2}} \\
& \left(\frac{1-x y+t x y+t x^{2} y}{1-t x-t^{2} x}-\frac{y(1+t+t x-x y)}{t^{2}-t y-y}\right)
\end{aligned}
$$

It is easy to see that

$$
\mathrm{CT} \frac{1}{1-3 x y-x^{2} y-x y^{2}} \frac{1-x y+t x y+t x^{2} y}{1-t x-t^{2} x}=\frac{1-x y}{1-3 x y-x^{2} y-x y^{2}},
$$

obtained by setting $t=0$, since it is a formal power series in $x$ and $y$, with coefficients in $\mathbb{Q}[t]$. Similarly,

$$
\mathrm{CT} \frac{y(1+t+t x-x y)}{t^{2}-t y-y}=\mathrm{CT} t^{-1} \frac{y\left(1+t^{-1}+x-t^{-1} x y\right)}{1-t^{-1} y-t^{-2} y}=0
$$

since it is a formal power series in $x$ and $y$ with coefficients in $t^{-1} \mathbb{Q}\left[t^{-1}\right]$.

Equation (48) then follows.

Similarly, we can compute the generating function of $\left(\begin{array}{c}i+j+r \\ 2 i-j\end{array}\right)$ for nonnegative integer $r$, from which it is easy to deduce (52).

\section{Example 9.3.}

$$
\sum_{i, j, r \geq 0}\left(\begin{array}{c}
i+j+r \\
2 i-j
\end{array}\right) x^{i} y^{j} z^{r}=\frac{1-z-x y(1-2 z-x z)}{\left(1-3 x y-x^{2} y-x y^{2}\right)\left(1-2 z+z^{2}-x z\right)} .
$$

Proof. We have

$$
\begin{aligned}
\sum_{i, j, r \geq 0}\left(\begin{array}{c}
i+j+r \\
2 i-j
\end{array}\right) x^{i} y^{j} z^{r} & =\sum_{i, j, r \geq 0}\left(\mathrm{CT}(1+t)^{2 i-j}\left(1+t^{-1}\right)^{2 j-i+u}\right) x^{i} y^{j} z^{r} \\
& =\mathrm{CT} \sum_{i, j, r \geq 0} \frac{(1+t)^{2 i}}{\left(1+t^{-1}\right)^{i}} x^{i} \cdot \frac{\left(1+t^{-1}\right)^{2 j}}{(1+t)^{j}} y^{j} \cdot\left(1+t^{-1}\right)^{r} z^{r} \\
& =\mathrm{CT} \frac{1}{1-t x-t x^{2}} \cdot \frac{1}{1-t^{-1} y-t^{-2} y} \cdot \frac{1}{1-z-t^{-1} z}
\end{aligned}
$$

By (76), this equals

$$
\mathrm{CT} \frac{1}{1-3 x y-x^{2} y-x y^{2}}\left(\frac{1-x y+t x y+t x^{2} y}{1-t x-t^{2} x}-\frac{y(1+t+t x-x y)}{t^{2}-t y-y}\right) \frac{1}{1-z-t^{-1} z} .
$$

Since $1 /\left(1-z-t^{-1} z\right)$ is a formal power series in $z$ with coefficients in $\mathbb{Q}\left[t^{-1}\right]$, we can discard the second part of (76) in our computation. So we have

$$
\mathrm{CT} \frac{1}{1-3 x y-x^{2} y-x y^{2}}\left(\frac{1-x y+t x y+t x^{2} y}{1-t x-t^{2} x}\right) \frac{1}{1-z-t^{-1} z} .
$$


Converting this into partial fraction in $t$, we get two parts, one with coefficients in $\mathbb{Q}[t]$, the other with coefficients in $t^{-1} \mathbb{Q}\left[t^{-1}\right]$. So we can discard the second part and set $t=0$ to get equation (77).

Algebraic Proof of Lemma 8.8. For $s$ with $1 \leq s \leq r$ and any $P \in \mathbb{A}_{s}(i, j)$, we factor $P$, according to its intersection with the line $y=0$, uniquely as $P_{1} P_{2}$, where $P_{1}$ is a $T^{(r)}$-path, and $P_{2}$ is an $H_{r}$ path starting with a vertical step. Then $S\left(P_{2}\right)=(k r+s, 0)$, for some $0 \leq k \leq j$. See the left picture of Figure 12 .
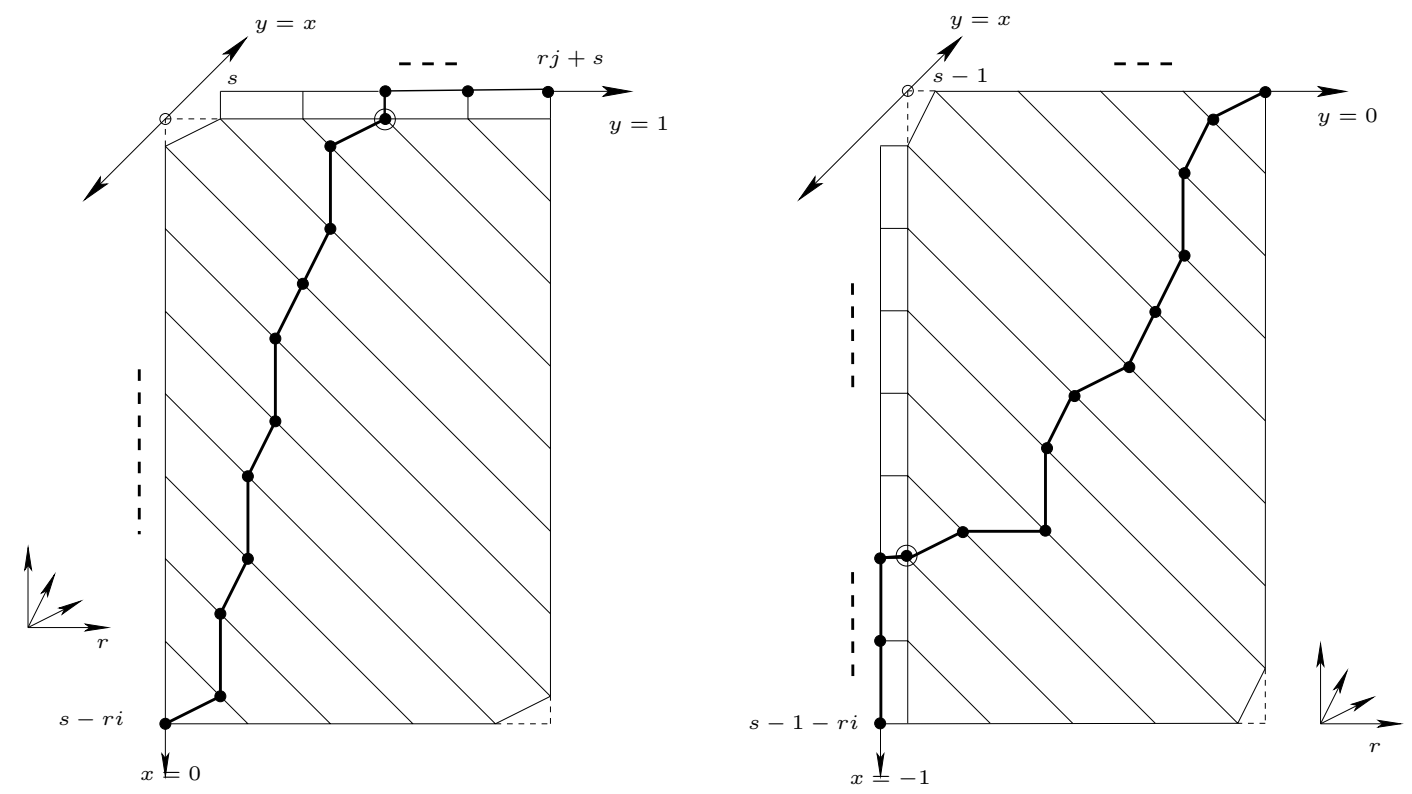

Figure 12: Example paths for $\mathbb{A}_{s}(i, j)$ and $\mathbb{B}_{s}(i, j)$.

For any $k$, there are $T^{(r)}(k r+s, i r-s)$ choices for $P_{1}$ since it is a $T^{(r)}$-path from $(0,-(i r-s))$ to $(k r+s, 0)$. There is only one choice for $P_{2}$ since it is a $(0,1)$ step followed by a fixed number of $(r, 0)$ steps. Conversely, any such $P_{1} P_{2}$ stays below the diagonal and hence belongs to $\mathbb{A}_{s}(i, j)$. Summing on all possible $k$, we get a formula for $\left|\mathbb{A}_{s}(i, j)\right|$ :

$$
\left|\mathbb{A}_{s}(i, j)\right|=\sum_{k=0}^{j} T^{(r)}(k r+s, i r-s) .
$$


Multiplying both sides of equation (78) by $x^{i} y^{j}$, and summing on $i$ and $j$, we have

$$
\begin{aligned}
\sum_{i, j=0}^{\infty}\left|\mathbb{A}_{s}(i, j)\right| x^{i} y^{j} & =\sum_{i, j=0}^{\infty} \sum_{k=1}^{i} T^{(r)}(k r+s, i r-s) x^{i} y^{j} \\
& =\sum_{i, j=0}^{\infty} \sum_{k=0}^{i}\left(\mathrm{CT} t^{s} \alpha^{k} \beta^{i}\right) x^{i} y^{j} \\
& =\mathrm{CT} t^{s} \sum_{j=0}^{\infty} \beta^{i} x^{i} \sum_{k=0}^{\infty} \alpha^{k} y^{k} \sum_{j \geq k} y^{j-k} \\
& =\mathrm{CT} t^{s} \frac{1}{(1-\alpha y)(1-\beta x)(1-y)}
\end{aligned}
$$

Similarly, any $Q \in \mathbb{B}_{s}(i, j)$ can be factored, according to its intersection with the line $x=0$, uniquely as $Q_{1} Q_{2}$, where $Q_{1}$ is a $V_{r}$ path ending with a horizontal step and $Q_{2}$ is a $T^{(r)}$-path. Then $E\left(Q_{1}\right)=\left(0,-\left(k^{\prime} r-s+1\right)\right)$ for some $1 \leq k^{\prime} \leq i$. See the right picture of Figure 12. We see that even in the case $s=1, k^{\prime}$ cannot be zero, since otherwise $Q_{1}$ will go above the diagonal.

For any $k^{\prime}$, there is only one choice for $Q_{1}$ since it is a fixed number of $(0, r)$ steps followed by a $(1,0)$ step. There are $T^{(r)}\left(j r+s-1, k^{\prime} r-s+1\right)$ choices for $Q_{2}$ since it is a $T^{(r)}$-path from $\left(0,-\left(k^{\prime} r-s+1\right)\right)$ to $(j r+s-1,0)$. Summing on all the possible $k^{\prime}$, we get a formula for $\left|\mathbb{B}_{s}(i, j)\right|$.

$$
\left|\mathbb{B}_{s}(i, j)\right|=\sum_{k^{\prime}=1}^{i} T^{(r)}\left(j r+s-1, k^{\prime} r-s+1\right) .
$$

A similar computation shows that

$$
\begin{aligned}
\sum_{i, j=0}^{\infty}\left|\mathbb{B}_{s}(i, j)\right| x^{i} y^{j} & =\mathrm{CT} t^{s-1} \sum_{j=0}^{\infty} \alpha^{j} y^{j} \sum_{k^{\prime}=1}^{\infty} \beta^{k^{\prime}} x^{k^{\prime}} \sum_{i \geq k^{\prime}} x^{i-k^{\prime}} \\
& =\mathrm{CT} t^{s-1} \frac{1}{1-\alpha y}\left(\frac{1}{1-\beta x}-1\right) \frac{1}{1-x} \\
& =\mathrm{CT} t^{s-1} \frac{1}{(1-\alpha y)(1-\beta x)(1-x)}-\mathrm{CT}^{s-1} \frac{1}{(1-\alpha y)(1-x)} \\
& =\mathrm{CT}^{s-1} \frac{1}{(1-\alpha y)(1-\beta x)(1-x)}-\delta_{s, 1} \frac{1}{(1-y)(1-x)} .
\end{aligned}
$$

To compute the two generating functions does not seem easy, but their difference has a simple form.

$$
\begin{aligned}
\mathrm{CT} t^{s} \frac{1}{1-\alpha y} \frac{1}{1-\beta x} \frac{1}{1-x}-\mathrm{CT}^{s-1} \frac{1}{1-\alpha y} & \frac{1}{1-\beta x} \frac{1}{1-y} \\
& =\mathrm{CT}^{s-1} \frac{t-t y-1+x}{(1-x)(1-y)(1-\alpha y)(1-\beta x)}
\end{aligned}
$$


Direct computation shows that

$$
\frac{t-t y-1+x}{(1-\alpha y)(1-\beta x)}=-\frac{x}{t^{r}(1-\beta x)}-\frac{1-y-t}{1-\alpha y}
$$

It is easy to see that $-\frac{1-y-t}{1-\alpha y}$, belonging to $\mathbb{Q}[t][[y]]$, has constant term constant term -1 in $t$, and $-\frac{x}{t^{r}(1-\beta x)}$, belonging to $t^{-1} \mathbb{Q}\left[t^{-1}\right][[x]]$, has constant term 0 . Hence

$$
\mathrm{CT} t^{s-1} \frac{t-t y-1+x}{(1-x)(1-y)(1-\alpha y)(1-\beta x)}=-\delta_{s, 1} \frac{1}{(1-x)(1-y)} .
$$

Put the above altogether, we obtain

$$
\sum_{i, j=0}^{\infty}\left(\left|\mathbb{A}_{s}(i, j)\right|-\left|\mathbb{B}_{s}(i, j)\right|\right) x^{i} y^{j}=0
$$

\section{Lemma 9.4.}

$$
\sum_{i, j \geq 0} T^{(r)}(r i, r j) x^{i} y^{j}=\frac{x(1-x)^{r-1}-y(1-y)^{r-1}}{x(1-x)^{r}-y(1-y)^{r}} .
$$

Proof. Let $x=u v$ and $y=v$. Then

$$
\begin{aligned}
\sum_{i, j \geq 0} T^{(r)}(r i, r j) x^{i} y^{j} & =\sum_{i, j \geq 0} T^{(r)}(r i, r j) u^{i} v^{i+j} \\
& =\sum_{n \geq i \geq 0} T^{(r)}(r i, r(n-i)) u^{i} v^{n} \\
& =\sum_{n \geq i \geq 0} \mathrm{CT} \frac{\alpha^{n}}{t^{r i}} u^{i} v^{n} \\
& =\mathrm{CT} \frac{1}{1-u t^{-r}} \frac{1}{1-\alpha v} .
\end{aligned}
$$


We have

$$
\begin{aligned}
\frac{1}{1-\alpha v} & =\frac{1-t}{(1-t)-v\left(1-t^{r+1}\right)} \\
& =\frac{1}{1-v} \frac{1-t}{1-t \frac{1-t^{r} v}{1-v}} \\
& =\frac{1}{1-v} \frac{(1-t)\left(1+t \frac{1-t^{r} v}{1-v}+\cdots+\left(t \frac{1-t^{r} v}{1-v}\right)^{r-1}\right)}{1-t^{r}\left(t \frac{1-t^{r} v}{1-v}\right)^{r}} \\
& =\frac{1}{1-v} \frac{1-t^{r}\left(\frac{1-t^{r} v}{1-v}\right)^{r-1}}{1-t^{r}\left(t \frac{1-t^{r} v}{1-v}\right)^{r}}+\text { other terms }
\end{aligned}
$$

Since the other terms contain only terms like $a_{r m+s} t^{r m+s}$ for $1 \leq s \leq r-1$, they do not contribute to the constant term in $t$. Let $z=t^{r}$. Then we have

$$
\mathrm{CT} \frac{1}{1-u t^{-r}} \frac{1}{1-\alpha v}=\mathrm{CT}_{z} \frac{1}{1-u z^{-1}} \frac{1}{1-v} \frac{1-z\left(\frac{1-z v}{1-v}\right)^{r-1}}{1-z\left(\frac{1-z v}{1-v}\right)^{r}}
$$

where $\mathrm{CT}_{z}$ means to take the constant term of a function in $z$. Since the other part of the right side of the above equation is a formal power series in $z$, it is straightforward to obtain

$$
\mathrm{CT} \frac{1}{1-u t^{-r}} \frac{1}{1-\alpha v}=\frac{1}{1-v} \frac{1-u\left(\frac{1-u v}{1-v}\right)^{r-1}}{1-u\left(\frac{1-u v}{1-v}\right)^{r}} .
$$

Replacing $u$ with $x / y$ and $v$ with $y$, we get formula (80)

\section{Theorem 9.5.}

$\operatorname{det}\left(g_{i+j}^{(r)}\right)_{0 \leq i, j \leq n-1}=\operatorname{det}\left(T^{(r)}(r i, r j)\right)_{0 \leq i, j \leq n-1}=\operatorname{det}\left(T^{(r-1)}(r j-i, r i-j)\right)_{0 \leq i, j \leq n-1}$.

Note that the identities in this theorem appeared in (72) and (73).

Proof. We use the technique of Section 4. The generating function for the first determinant is

$$
\frac{x g^{(r)}(x)-y g^{(r)}(y)}{x-y} .
$$


Since $f^{(r)}(x)=g^{(r)}(x)-1$ is a formal power series in $x$ satisfying

$$
f^{(r)}(x)=x /\left(1+f^{(r)}(x)\right)^{r+1}
$$

we make the substitution $x \rightarrow x /(1+x)^{r+1}$ and $y \rightarrow y /(1+y)^{r+1}$. The generating function becomes

$$
\frac{x(1+x)^{-r-1}(1+x)-y(1+y)^{-r-1}(1+y)}{x(1+x)^{-r-1}-y(1+y)^{-r-1}}=\frac{x(1+x)^{-r}-y(1+y)^{-r}}{x(1+x)^{-r-1}-y(1+y)^{-r-1}} .
$$

Normalizing and dividing by $(1+x)(1+y)$, we get

$$
\frac{x(1-x)^{r-1}-y(1-y)^{r-1}}{x(1-x)^{r}-y(1-y)^{r}} .
$$

The first equality hence follows from Lemma 9.4.

To show the second equality, we compute the generating function for the third determinant.

$$
\begin{aligned}
\sum_{i, j \geq 0} T^{(r-1)} & (r j-i, r i-j) x^{i} y^{j} \\
& =\sum_{i, j \geq 0} T^{(r-1)}((r-1) j+(j-i),(r-1) i-(j-i)) x^{i} y^{j} \\
& =\sum_{i, j \geq 0} \mathrm{CT} t^{j-i}\left(1+t+\cdots+t^{r-1}\right)^{j}\left(1+t^{-1}+\cdots+t^{-r+1}\right)^{i} x^{i} y^{j} \\
& =\mathrm{CT} \sum_{i, j \geq 0}\left(t^{-1}+t^{-2} \cdots+t^{-r}\right)^{i} x^{i}\left(t+t^{2}+\cdots+t^{r}\right)^{j} y^{j} \\
& =\mathrm{CT} \frac{1}{1-\left(t^{-1}+t^{-2} \cdots+t^{-r}\right) x} \frac{1}{1-\left(t+t^{2}+\cdots+t^{r}\right) y}
\end{aligned}
$$

Similarly, the generating function for the second determinant is

$$
\mathrm{CT} \frac{1}{1-\left(1+t^{-1}+t^{-2} \cdots+t^{-r}\right) x} \frac{1}{1-\left(1+t+t^{2}+\cdots+t^{r}\right) y} .
$$

The following computation shows that the (83) can be obtained from (82) by making the substitution $x \rightarrow x /(1-x)$ and $y \rightarrow y /(1-y)$, and then dividing by $(1-x)(1-y)$. This yields the second equality.

$$
\begin{aligned}
& \mathrm{CT} \frac{1}{1-\left(1+t^{-1}+t^{-2} \cdots+t^{-r}\right) x} \frac{1}{1-\left(1+t+t^{2}+\cdots+t^{r}\right) y} \\
& =\mathrm{CT} \frac{1}{1-x-\left(t^{-1}+t^{-2} \cdots+t^{-r}\right) x} \frac{1}{1-y-\left(t+t^{2}+\cdots+t^{r}\right) y} \\
& =\mathrm{CT} \frac{1}{(1-x)(1-y)} \frac{1}{1-\left(t^{-1}+t^{-2} \cdots+t^{-r}\right) \frac{x}{1-x}} \frac{1}{1-\left(t+t^{2}+\cdots+t^{r}\right) \frac{y}{1-y}}
\end{aligned}
$$




\section{References}

[1] M. Abramowitz and I. A. Stegun, Handbook of Mathematical Functions, Dover, New York, 1972.

[2] G. E. Andrews, Plane partitions. V. The TSSCPP conjecture, J. Combin. Theory Ser. A 66 (1994), 28-39.

[3] G. E. Andrews and W. H. Burge, Determinant identities, Pacific J. Math. 158 (1993), $1-14$.

[4] É. Barbier, Généralisation du problème résolu par M. J. Bertrand, C. R. Acad. Sci. Paris 105 (1887), 407.

[5] D. M. Bressoud, Proofs and Confirmations: The Story of the Alternating Sign Matrix Conjecture. Cambridge University Press, 1999.

[6] Ö. Ĕ̆gecioğlu, T. Redmond, and C. Ryavec, From a polynomial Riemann hypothesis to alternating sign matrices, Electron. J. Combin. 8 (2001), no. 1, R36, 51 pp.

[7] G. P. Egorychev, Integral Representation and the Computation of Combinatorial Sums, Translations of Mathematical Monographs, vol. 59, American Mathematical Society, Providence, RI, 1984.

[8] I. M. Gessel, Solution to part (B) of Problem 6151, Partitions of finite sets, Amer. Math. Monthly 86 (1979), 64-65. Proposed by Clarence H. Best, Amer. Math. Monthly 84 (1977), 391.

[9] _ A factorization for formal Laurent series and lattice path enumeration, J. Combin. Theory Ser. A 28 (1980), 321-337.

[10] _ A probabilistic method for lattice path enumeration, J. Statist. Plann. Inference 14 (1986), 49-58.

[11] I. M. Gessel and G. Viennot, Binomial determinants, paths, and hook length formulae, Adv. Math. 58 (1985), 300-321.

[12] I. P. Goulden and L. G. Serrano, Maintaining the spirit of the reflection principle when the boundary has arbitrary integer slope, J. Combin. Theory Ser. A 104 (2003), 317-326.

[13] I. P. Goulden and D. M. Jackson, Combinatorial Enumeration, Dover Publications Inc., Mineola, NY, 2004. Reprint of the 1983 original.

[14] W. B. Jones and W. J. Thron, Continued Fractions: Analytic Theory and Applications, Encyclopedia of Mathematics and its Applications, vol. 11, Addison-Wesley, Reading, MA, 1980.

[15] C. Krattenthaler, An alternative evaluation of the Andrews-Burge determinant, Mathematical Essays in Honor of Gian-Carlo Rota (Cambridge, MA, 1996), Progr. Math., vol. 161, Birkhäuser Boston, Boston, MA, 1998, pp. 263-270.

[16] _ Advanced determinant calculus, Séminaire Lotharingien Combin. 42 (1999), Article B42q, 67 pp. 
[17] _ Advanced determinant calculus: A complement, Linear Algebra Appl. 411 (2005), 68-166.

[18] G. Kuperberg, Another proof of the alternating-sign matrix conjecture, Internat. Math. Res. Notices (1996), 139-150.

[19] _ Symmetry classes of alternating-sign matrices under one roof, Ann. of Math. (2) 156 (2002), 835-866.

[20] W. Lang, On generalizations of the Stirling number triangles, J. Integer Seq. 3 (2000), no. 2, Article 00.2.4.

[21] W. H. Mills, D. P. Robbins, and H. Rumsey, Jr., Alternating sign matrices and descending plane partitions, J. Combin. Theory Ser. A 34 (1983), 340-359.

[22] _ Enumeration of a symmetry class of plane partitions, Discrete Math. 67 (1987), 43-55.

[23] S. Okada, Enumeration of symmetry classes of alternating sign matrices and characters of classical groups, arXiv:math.CO/0408234.

[24] M. Petkovšek and H. S. Wilf, A high-tech proof of the Mills-Robbins-Rumsey determinant formula, Electron. J. Combin. 3 (1996), no. 2, R19, 3 pp.

[25] D. P. Robbins, The story of 1, 2, 7, 42, 429, 7436, $\cdots$, Math. Intelligencer 13 (1991), no. $2,12-19$.

[26] _ Symmetry classes of alternating sign matrices, arXiv:math.CO/0008045.

[27] M. Somos, Number walls in combinatorics, http://cis.csuohio.edu/ somos/nwic.html, September 20, 2000.

[28] U. Tamm, Some aspects of Hankel matrices in coding theory and combinatorics, Electron. J. Combin. 8 (2001), no. 1, A1, 31 pp.

[29] J. West, Sorting twice through a stack, Theoret. Comput. Sci. 117 (1993), no. 1-2, 303-313, Conference on Formal Power Series and Algebraic Combinatorics (Bordeaux, 1991).

[30] D. Zeilberger, A proof of Julian West's conjecture that the number of two-stacksortable permutations of length $n$ is $2(3 n) ! /((n+1) !(2 n+1) !)$, Disc. Math. 102 (1992), 85-93.

[31] _ Proof of the alternating sign matrix conjecture, The Foata Festschrift, Electron. J. Combin. 3 (1996), no. 2, R13, 84 pp. 